\title{
Exploitation des invertébrés par les sociétés précolombiennes des Petites Antilles
}

Nathalie Serrand

\section{OpenEdition}

12 Journals

Édition électronique

URL : https://journals.openedition.org/jsa/6213

DOI : $10.4000 /$ jsa.6213

ISSN : 1957-7842

Éditeur

Société des américanistes

Édition imprimée

Date de publication : 1 décembre 2007

Pagination : 7-47

ISSN : 0037-9174

\section{Référence électronique}

Nathalie Serrand, «Exploitation des invertébrés par les sociétés précolombiennes des Petites Antilles ", Journal de la Société des américanistes [En ligne], 93-1 | 2007, mis en ligne le 15 juin 2012, consulté le 03 septembre 2022. URL : http://journals.openedition.org/jsa/6213 ; DOI : https://doi.org/ $10.4000 /$ jsa. 6213 


\title{
EXPLOITATION DES INVERTÉBRÉS PAR LES SOCIÉTÉS PRÉCOLOMBIENNES \\ DES PETITES ANTILLES
}

\author{
Nathalie SERRAND *
}

Les invertébrés (mollusques et crustacés) ont été des ressources non négligeables pour les sociétés précolombiennes présentes dans les Petites Antilles entre le $\mathrm{VI}^{\circ}$ millénaire av. J.-C. et le $x v^{\mathrm{e}}$ siècle apr. J.-C. Leur prise en compte par le biais des analyses archéozoologiques contribue à définir les systèmes socio-économiques et culturels de ces sociétés. Les données de vingt-et-un sites répartis sur sept îles des Petites Antilles permettent d'esquisser l'évolution des schémas d'exploitation durant les phases chrono-culturelles majeures. Les mollusques semblent intervenir dans la mobilité et le mode d'occupation des espaces des premiers occupants, chasseurs-cueilleurs, de la période archaïque. L'exploitation des invertébrés par les premiers groupes sédentaires du début de la période céramique présente des spécificités qui suggèrent une économie homogène, peu diversifiée localement, reflet d'une relative cohésion culturelle. Durant la suite de la période céramique, les formes d'exploitation, plus variées, sont marquées par des changements de cibles qui suggèrent une gestion plus pragmatique et intensive de ces ressources, dans un contexte de diversification culturelle. Ces grandes tendances doivent encore être affinées par la meilleure compréhension du cadre paléoenvironnemental et des systèmes techniques de ces sociétés. [Mots-clés : archéozoologie, Antilles précolombiennes, invertébrés marins et terrestres, économie de subsistance, système technique.]

Exploitation of invertebrates by the Precolumbian societies of the Lesser Antilles. Invertebrates (molluscs and crustaceans) were significant resources for the Precolumbian societies present in the West Indian islands between the VIth millenium B.C. and the xvth century A.D. Integrating them through archaeozoological analysis contributes to the definition of the socio-economical and cultural systems of these societies. Data derived from twenty one sites distributed on seven islands of the Lesser Antilles allow to sketch the evolution of the exploitation patterns during the major chronocultural phases. Molluscs seem to have played a role in the mobility and territorial

* CNRS/Muséum national d'Histoire naturelle, « Écologie et gestion de la biodiversité, archéozoologie, histoire des sociétés humaines et des peuplements animaux » (UMR 5197), Bâtiment d'anatomie comparée, 55 rue Buffon, 75005 Paris [serrand@mnhn.fr].

Journal de la Société des Américanistes, 2007, 93-1, pp. 7-47. OSociété des Américanistes. 
occupation patterns of the first hunter-gatherer settlers of the Archaic period. Exploitation of invertebrates by the first sedentary groups of the early Ceramic period, shows specificities which suggest an homogenous economy, little diversified locally, reflecting a relative cultural cohesion. During the rest of the Ceramic period, the exploitation patterns appear more varied. They are characterized by changes in targets which suggest a more pragmatic and intensive managment of the resources, in a context of cultural diversification. These main trends still need to be refined with a better understanding of the paleo-environmental frame and the technical systems of these societies. [Key words : archaeozoology, Precolumbian Antilles, marine and terrestrial invertebrates, subsistance economy, technical system.]

Aprovechamiento de los invertebrados por las sociedades prehispánicas de las Antillas Menores. Invertebrados (molluscos y crustáceos) fueron recursos no despreciables para las sociedades precolombinas presentes en las Antillas Menores entre de el VIo milenio a.C. y el siglo xv d.C. Su integración mediante los análisis arqueozoológicos contribuye a la definición de los sistemas socioeconómicos y culturales de estas sociedades. Los datos de veintiún sitios distribuidos en siete islas de las Antillas Menores permiten esbozar la evolución de los esquemas de explotación durante las fases crono-culturales principales. Molluscos parecen intervenir en la movilidad y el modo de ocupación de los espacios de los primeros ocupantes, cazadores y colectores del período arcaico. La explotación de los invertebrados por los primeros grupos sedentarios del principio del período cerámico, presenta especificidades que sugieren una economía homogénea, poco diversificada localmente, reflejo de una relativa cohesión cultural. Durante el resto del período cerámico, las formas de explotación, más variadas, se caracterizan por cambios de objetivos que sugieren una gestión más pragmática y más intensiva de estos recursos, en un contexto de diversificación cultural. Estas grandes tendencias deben aún ser precisadas por la mejor comprensión del marco medioambiental y de los sistemas técnicos de estas sociedades. [Palabras claves : arqueozoología, Antillas precolombinas, invertebrados marinos y terrestres, economía de subsistencia, sistemas técnicos.]

\section{INTRODUCTION}

Les organismes invertébrés, essentiellement les mollusques et crustacés, ont joué un rôle non négligeable dans les économies de subsistance et de production artisanale des sociétés précolombiennes présentes dans les îles des Antilles entre le $\mathrm{VI}^{\mathrm{e}}$ millénaire av. J.-C. et le $\mathrm{xv}^{\mathrm{e}}$ siècle de notre ère (Figure 1). L'étude archéozoologique et technologique des restes d'invertébrés provenant des sites datés de cet intervalle contribue donc à esquisser l'histoire des relations entre les populations amérindiennes et les espaces naturels insulaires et à comprendre leurs systèmes socio-économiques et culturels.

La première occupation des îles des Antilles, à partir du $\mathrm{VI}^{\mathrm{e}}$ millénaire av. J.-C. (Figure 1), est le fait de groupes de chasseurs-cueilleurs vraisemblablement non sédentaires (période archaïque) dont les origines continentales et l'organisation socio-économique sont peu connues (Kozlowski 1980 ; Veloz 


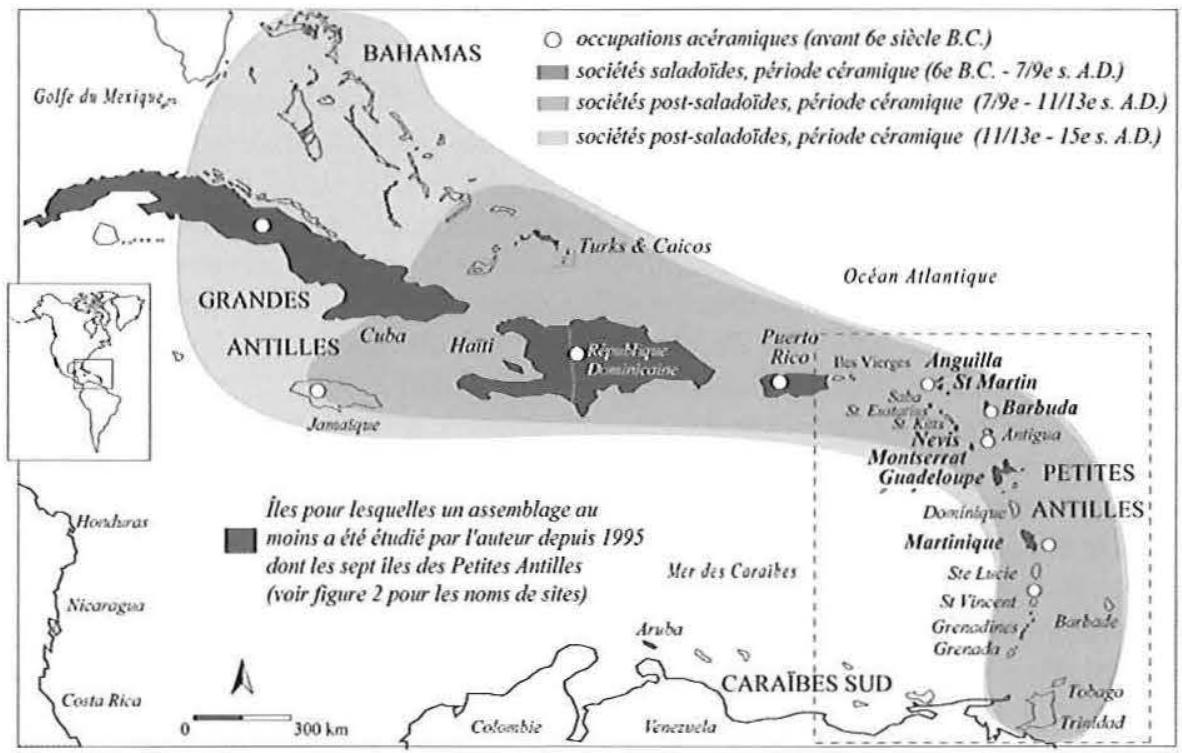

Fig. 1 - Îles des Antilles au sein de la zone Caraïbe, schéma des grandes phases d'occupation précolombienne et localisation des îles oủ des assemblages ont été étudiés (voir Figures 2 et 3).

Maggiolo et Vega 1982 ; Rouse 1992 ; Keegan 1994). La seconde phase majeure de colonisation (Figure 1), à partir du vle siècle av. J.-C., correspond à l'installation de sociétés d'agriculteurs potiers (période céramique), sédentaires, originaires du Venezuela et des Guyanes (Rouse et Cruxent 1963; Rouse 1986, 1992 ; Keegan 2000). Ces sociétés occupent initialement un espace circonscrit à la portion orientale des Antilles (Figure 1) dans une relative homogénéité culturelle qui perdure jusque vers les $\mathrm{VII}^{\mathrm{e}}$ et IX $\mathrm{IX}^{\mathrm{e}}$ siècles apr. J.-C. À partir de cette période et jusqu'au $\mathrm{Xv}^{\mathrm{c}}$ siècle, émerge une mosaïque culturelle complexe (Figure 1) résultant d'évolutions locales et de mouvements et influences entre les îles et le continent dans un espace progressivement élargi à l'ensemble des Grandes et Petites Antilles et des Bahamas (voir synthèses in Rouse 1992 ; Keegan 2000 ; Delpuech et Hofman 2004).

Les économies de subsistance des sociétés antillaises des périodes archaïque et céramique combinaient la chasse, la pêche et la collecte de ressources animales et végétales, terrestres et marines, autochtones, mais également introduites depuis le continent ou d'une île à une autre (DeFrance et al. 1996 ; Petersen 1997 ; Newsom et Wing 2004). Ces ressources complétaient, durant la période céramique, les productions vivrières horticoles, voire agricoles, centrées largement sur la culture du manioc (introduit depuis le continent), mais aussi d'arbres fruitiers, d'herbes 
panifiables et d'essences alimentaires ou médicinales (Meggers et Evans 1978; Ortiz Aguilu et al. 1991 ; Newsom 1993 ; DeFrance et al. 1996 ; Newsom et Wing 2004). Au sein de ces composantes, les invertébrés marins et terrestres ont joué un rôle non négligeable, en particulier les mollusques qui constituaient à la fois une ressource alimentaire et un matériau de production d'outils et d'éléments de parure. Ce rôle prend d'autant plus d'importance si l'on considère la faible diversité de la faune terrestre, notamment mammalienne (dépourvue de taxons de grande taille, vulnérable aux pressions de prédation), et la répartition inégale des matières premières (des gîtes de silex ne sont, par exemple, attestés que sur trois îles : Knippenberg 2006) caractéristiques des îles antillaises.

Des variations de l'importance des différentes ressources terrestres et marines, dont les mollusques et les crustacés, sont observables au long de la séquence d'occupation précolombienne des Petites Antilles. Les mollusques, en particulier, semblent avoir constitué une composante importante des économies de subsistance des groupes de la période archaïque et pourraient avoir eu un rôle dans leur mobilité et leur mode d'occupation des espaces. Ce rôle, s'il paraît moins prépondérant dans les économies de subsistance de la période céramique, reste toutefois non négligeable et accompagne certaines des évolutions socio-économiques des sociétés d'agriculteurs de cette période. Ces variations au cours du temps reflètent en partie les facteurs environnementaux, sociaux, démographiques et économiques qui sont intervenus dans l'évolution des sociétés précolombiennes antillaises (Petersen 1997; Newsom et Wing 2004). La compréhension de ces variations, bien que délicate, peut donc permettre de mieux appréhender, outre les modes de vie de ces populations, l'histoire de leur implantation et de leur évolution culturelle et socio-économique.

La perception de ces formes d'économies et de leur évolution dans l'espace et le temps nécessite l'analyse systématique d'assemblages de restes fauniques issus de contextes géographiques et archéologiques variés et bien documentés et l'étude des processus environnementaux intervenant en parallèle. C'est ce qui a été entrepris avec un corpus de vingt-et-un sites (Figure 2) couvrant la séquence d'occupation précolombienne, localisés sur sept îles des Petites Antilles (Figure 3). Ces données s'ajoutent aux informations issues d'autres travaux, pour lesquelles, toutefois, des problèmes de comparaison se posent du fait d'approches analytiques différentes. Si, dans l'état des données, il n'est pas possible de produire une synthèse à l'échelle de l'archipel, quelques lignes de réflexion générales peuvent cependant être ébauchées sur l'évolution de l'exploitation des invertébrés durant l'occupation précolombienne des Petites Antilles et sur leur portée pour la compréhension des systèmes économiques et des changements techno-culturels au cours du temps. 


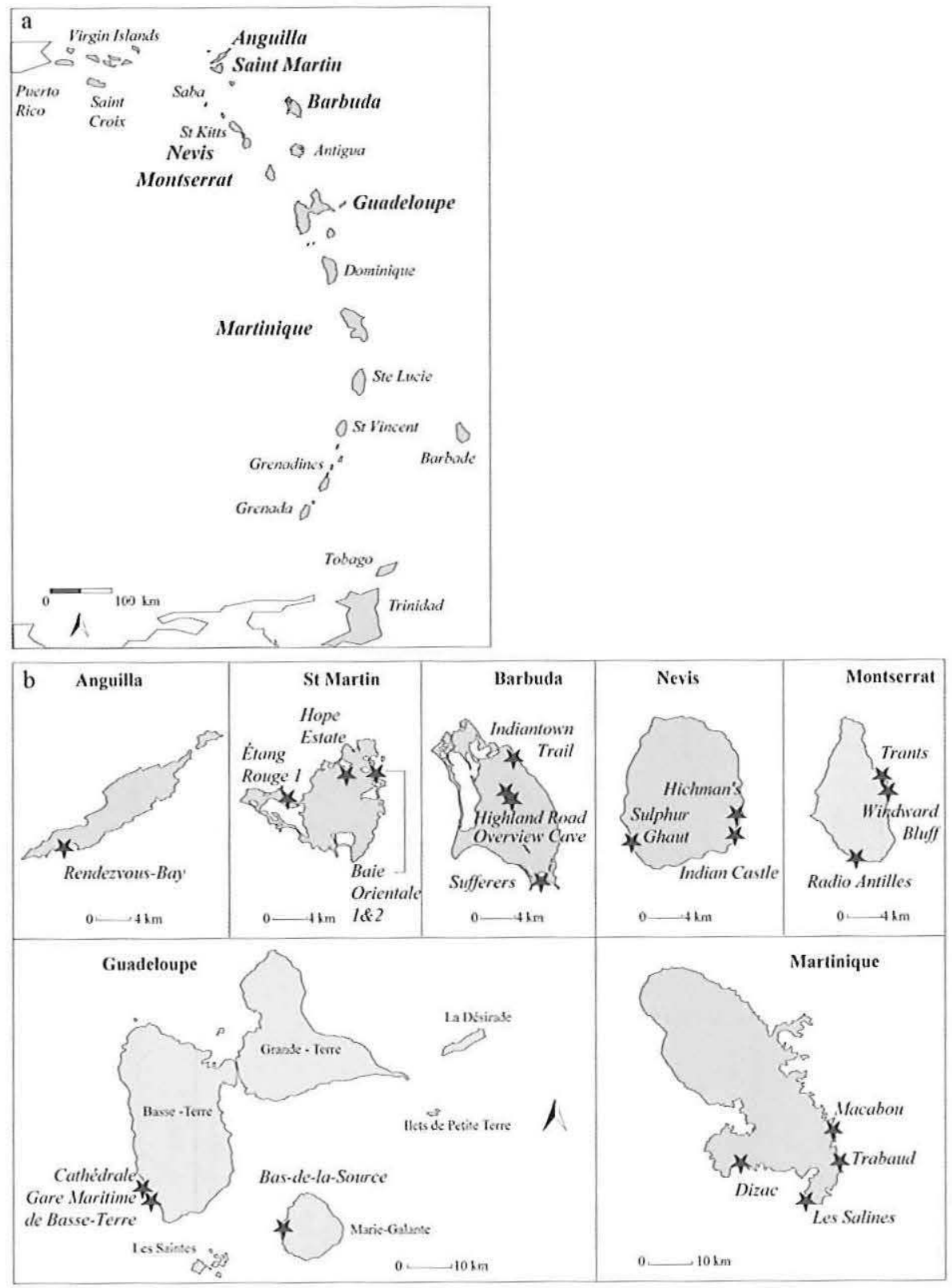

FIG. 3 - a. Carte des Petites Antilles avec, en gras, les îles dont les sites sont présentés dans le texte ; b. Détail des îles (du nord au sud) et localisation des sites étudiés. 


\section{Matériels et MÉTHOdes}

Les données (Figure 2) représentent un corpus totalisant plus de 300000 restes issus de vingt-et-un sites répartis sur les sept îles suivantes: Anguilla, Saint-Martin, Barbuda, Nevis, Montserrat, Guadeloupe et Martinique (Figure 3). Quelques sites supplémentaires étudiés par l'auteur à Puerto Rico et dans les Bahamas (Figure 1) sortent du cadre des Petites Antilles et ne seront que brièvement mentionnés.

Le corpus a été étudié de plusieurs points de vue, taxinomique, taphonomique, statistique et technique en articulant les informations sur la consommation alimentaire et le travail de la coquille (Serrand 2002). L'analyse des assemblages de sites, menée selon une démarche similaire adaptée en fonction des effectifs, a permis de caractériser l'exploitation des invertébrés sur chacun des sites, tout en constituant un corpus de données comparables. Les cortèges d'espèces et les milieux exploités ont été définis en tenant compte des observations taphonomiques déterminant la représentation archéologique des taxons. Les variations stratigraphiques des spectres ont été analysées pour chaque site. Enfin, les éléments se rapportant au travail de la coquille ont été caractérisés du point de vue typo-technologique : les pièces modifiées pour la production d'objets, quelquesunes des techniques de production et leur enchaînement en chaînes opératoires ont été décrits.

La comparaison des résultats permet d'évaluer les constantes et les différences entre les assemblages : représentation des taxons et des milieux exploités ; récurrences dans la taphonomie des restes; utilisation des taxons pour le travail de la coquille ; stratégies de collecte dans les différents milieux naturels, etc. Dans la mesure du possible, les données sur l'exploitation des autres ressources fauniques, essentiellement de vertébrés, ont été intégrées quand elles existaient. La confrontation de l'ensemble de ces données permet d'esquisser quelques lignes de réflexion sur l'évolution de l'exploitation des invertébrés durant l'occupation précolombienne des Petites Antilles.

\section{ÉVOLUTION DIACHRONIQUE DES SCHÉMIAS D'EXPLOITATION DES INVERTÉBRÉS}

L'exploitation des mollusques: une activité clé dans la gestion territoriale des groupes non sédentaires de chasseurs-cueilleurs de la période archä̈que

Pour les Petites Antilles, les sites de l'âge archaïque, situés entre le $\mathrm{III}^{\mathrm{e}}$ et le $\mathrm{I}^{\mathrm{er}}$ millénaires av. J.-C., sont essentiellement côtiers : les plus anciens sont Jolly Beach à Antigua (Davis 1982 ; Nodine 1990) et Norman Estate à Saint-Martin (Brokke 1999; Knippenberg 1999) ainsi que River Site à Barbuda (Watters 2001) et Whitehead's Bluff à Anguilla (Crock et al. 1995) qui pourraient correspondre 
à une phase archaïque ancienne (Hofman et al. 2006). Viennent ensuite Krum Bay à Saint Thomas (Bullen 1973; Lundberg 1989), Corre Corre Bay à SaintEustache (Versteeg et al. 1993), Sugar Factory Pier à Saint-Kitts (Armstrong 1978 ; Goodwin 1978), Hichman's Shell Heap à Nevis (Wilson 1989) et Pointe des Pies en Guadeloupe (Richard 1994), peut-être représentatifs d'une phase archaïque récente. Trois sites récemment fouillés complètent cette liste et ajoutent d'importants jalons dans la connaissance des modes de vie des groupes archaïques des Petites Antilles : celui de Plum Piece, unique site connu de l'intérieur des terres, localisé à Saba et daté entre 1875 et 1520 cal. av. J.-C. (Hofman et Hoogland 2003; Hofman et al. 2006) ; et, sur l'île de Saint-Martin (Figure 3), les sites de Baie Orientale 1 et Étang Rouge 1 étudiés sous la direction de Dominique Bonnissent de l'INRAP Guadeloupe (Bonnissent et al. 2001, 2002a, 2005 ; Figure 2). Le premier est datéentre 800 et $400 \mathrm{cal}$. av. J.-C.et est exceptionnellement bien préservé,notamment dans son organisation spatiale ; le second, plus ancien, présente une datation dans la partie médiane de la stratigraphie de $3490 \pm 40$ B.P.

Dans l'état des données, leurs outillages ne sont pas attribués avec certitude à une des deux séries archaïques. Les assemblages fauniques de ces gisements, étudiés ici, permettent d'observer des schémas d'occupation de site et d'exploitation des ressources en grande partie centrés sur les mollusques, pour la consommation et, sur le site de Baie Orientale 1, pour la production d'outils.

Sur le gisement de Baie Orientale 1 (Serrand et Bonnissent 2005), l'assemblage faunique ne comprend que 11 individus de poissons et 65 de crustacés et est largement dominé par les mollusques ( 23198 restes ; 4550 individus). La collecte a été ciblée sur cinq taxons majeurs (Figure 4a), le lambi Strombus gigas, les nérites versicolor et peloronta, le burgo Cittarium pica et le chiton Acanthopleura gramulata, accessibles à proximité du site, dans les herbiers infralittoraux des baies avoisinantes et sur le médiolittoral rocheux les séparant.

Cette collecte apparaît pragmatique puisque, d'une part, les espèces les plus grandes parmi celles accessibles dans les zones les moins profondes ont été privilégiées : c'est le cas des espèces de nérites peloronta et versicolor plus grandes que leur voisine $N$. tessellata, ou du chiton Acanthopleura granulata plus grand que les autres chitons avec lesquels il partage les zones rocheuses. Par ailleurs, pour les deux taxons majeurs Cittarium pica et Strombus gigas, ce sont les individus de taille petite à moyenne qui ont été privilégiés parce qu'ils sont les plus accessibles (Stoner et Waite 1990). Les occupants du gisement de Baie Orientale 1 ont donc exploité les mollusques de manière pragmatique en privilégiant les zones d'accès aisé, suffissamment productives. La collecte de la majorité de ces taxons a eu comme finalité la consommation alimentaire. Les différentes espèces étaient cuites dans leur coquille, par exposition indirecte à la chaleur, sur des pierres chauffées organisées en aires de cuisson, traitement culinaire orginal mis en évidence pour la première fois (Bonnissent et al. 2001, 2002a ; Serrand et Bonnissent 2005). La collecte à Baie Orientale 1 concernait, toutefois, aussi des 


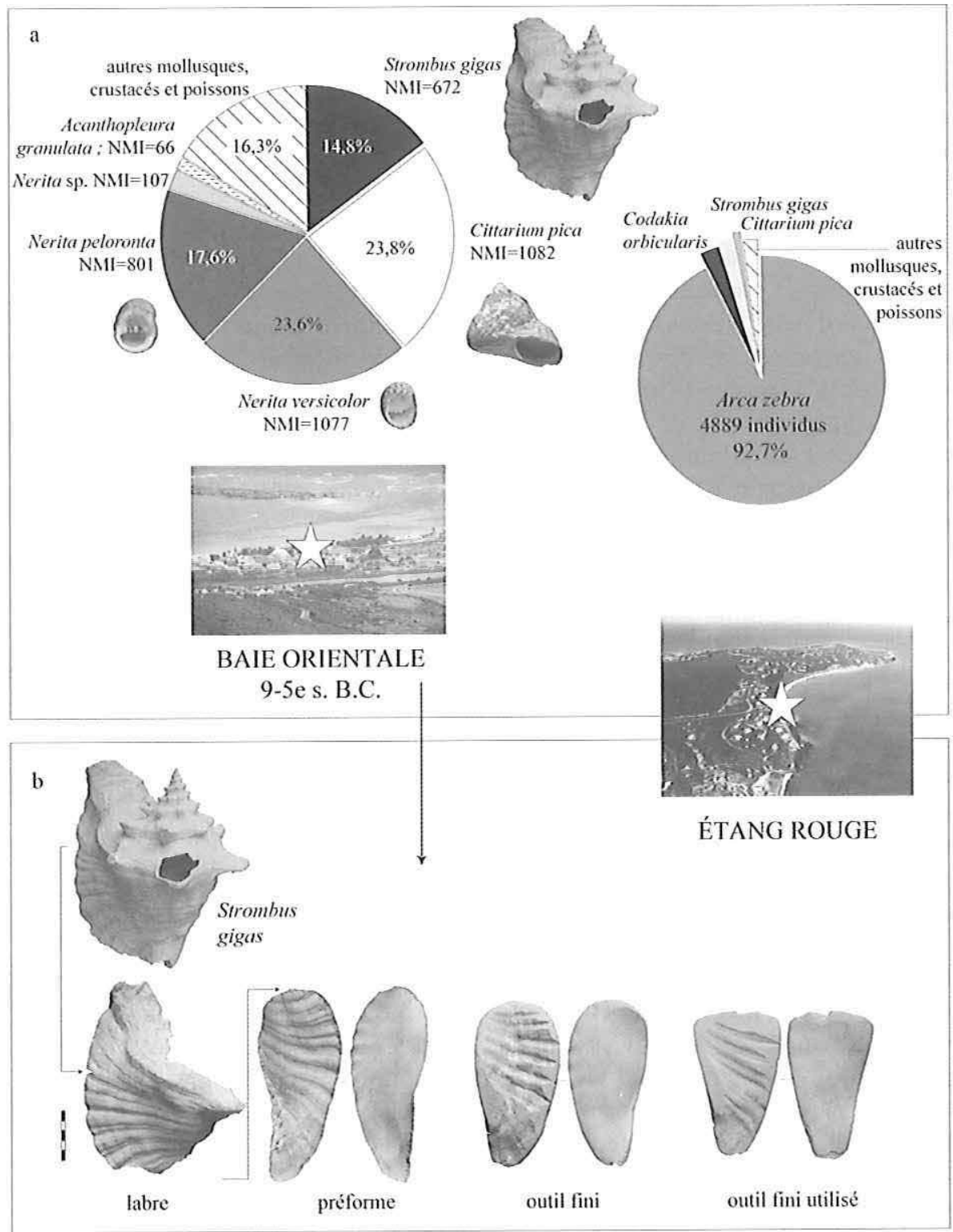

FIG. 4 - a. Fréquences relatives d'individus ( $\%$ NMI) des espèces fauniques majeures exploitées sur les sites de Baie Orientale 1 et Étang Rouge 1 à Saint-Martin ; b. Étapes de production des outils tranchants sur labres de Strombus gigas de Baie Orientale 1. 
individus adultes du lambi Strombus gigas qui, tout en étant consommés, étaient recherchés spécifiquement pour leurs labres épais, supports d'une production intensive et standardisée d'outils tranchants probablement voués à la fabrication d'embarcations. La chaîne opératoire de transformation des labres en outils est intégralement représentée sur le gisement (Figure 4b), de l'acquisition de la matière à l'utilisation des outils finis, incluant l'ensemble des déchets de production (Serrand et Bonnissent 2005). Le caractère systématique et standardisé de cette industrie, son inscription dans l'organisation spatiale du site, la quantité élevée de pièces produites (près de 200, stockées pour certaines) et sa perpétuation au travers d'occupations vraisemblablement ponctuelles, mais répétées, suggèrent qu'il s'agissait d'une des activités majeures ayant motivé l'occupation de Baie Orientale 1. L'accès à la matière première a donc sans doute été prépondérante et peut avoir déterminé, en partie, la localisation de ce site. La collecte à des fins alimentaires aurait alors été organisée secondairement en fonction de cette activité principale, par la mise à profit des taxons les plus aisément accessibles dans les milieux environnants.

À Étang Rouge 1 (Bonnissent et al. 2005; Serrand 2005a), l'assemblage faunique est également presque dépourvu de vertébrés et de crustacés et est dominé par les mollusques (14 026 restes ; 5260 individus). Il révèle, comme à Baie Orientale 1, une sélection étroite d'espèces (Figure 4a), cette fois-ci centrée sur un large bivalve, Arca zebra, ciblé (93\% NMI) de manière intensive et dont les bancs ont vraisemblablement été "ratissés ". Les spécimens étaient également préparés sur place dans des structures de cuisson moins élaborées qu'à Baie Orientale 1. La production d'industrie était, en revanche, très limitée. La quantité de spécimens consommés d'Arca zebra, la variété des classes d'âges et le rendement en biomasse suggèrent que son exploitation intensive à des fins alimentaires peut avoir été l'activité majeure liée à l'occupation d'Étang Rouge 1 et pourrait en avoir influencé la localisation.

Si les assemblages de Baie Orientale 1 et d'Étang Rouge 1 diffèrent, ils révèlent tous les deux l'exploitation pragmatique et intensive de populations riches d'une ou deux espèces ciblées de grande taille à des fins strictement alimentaires ou à dominante artisanale. Ces résultats, combinés aux autres données archéologiques, indiquent que ces activités d'exploitation de ressources locales ont déterminé, en partie au moins, l'installation de ces occupations. Le caractère spécialisé des activités menées sur chacun des sites et leur maintien au travers d'une fréquentation vraisemblablement courte, mais répétée, suggèrent une gestion très organisée des ressources, éventuellement selon un rythme saisonnier (Bonnissent et al. 2002a ; Serrand et Bonnissent 2005).

Ces résultats renforcent quelques tendances observées sur les autres sites du Nord des Petites Antilles de la période archaïque cités précédemment (Bullen 1973 ; Davis 1982 ; Armstrong 1978 ; Goodwin 1978 ; Lundberg 1989 ; Wilson 1989 ; Nodine 1990 ; Crock et al. 1995 ; Brokke 1999; Hofman et Hoogland 
2003). Bien que la disparité des méthodes de fouille et d'étude des faunes ne permette pas d'élaborer une vraie synthèse sur l'exploitation des mollusques, quelques aspects récurrents apparaissent malgré tout.

Les mollusques marins sont ainsi abondants et constituent la grande majorité des restes fauniques dans la plupart des sites. Les vertébrés et crustacés sont rares, excepté à Plum Piece, le seul site de l'intérieur des terres, qui en a livré de grandes quantités (Hofman et Hoogland 2003). Leur faible nombre pourrait résulter de biais de conservation différentielle ou de tamisage, mais cette dernière hypothèse est à exclure dans les cas de Baie Orientale 1 et Étang Rouge 1 où les sédiments ont été tamisés sur mailles de 4 à $10 \mathrm{~mm}$. La rareté des vertébrés et crustacés paraît bien indiquer une réelle prédominance des mollusques marins, avec des variantes peut-être liées aux spécificités fonctionnelles de chaque occupation et à leur zone d'implantation (côte/intérieur des terres).

L'exploitation des mollusques, sur la majorité des sites dont Baie Orientale 1 et Étang Rouge 1, est, par ailleurs, souvent intensive et ciblée sur quelques taxons et milieux, aisément accessibles en eaux peu profondes, avec une sélection d'individus parmi les plus accessibles et/ou rentables. Les espèces majeures sélectionnées sont assez récurrentes : ce sont souvent de grands bivalves de fonds rocheux, coralliens et sableux comme à Norman Estate (Brokke 1999), Sugar Factory Pier (Armstrong 1978; Goodwin 1978) ou Jolly Beach (Davis 1982; Nodine 1990) où Arca zebra domine (plus de $60 \%$ NMI à Norman Estate ; $50 \%$ NMI à Jolly Beach), devant d'autres bivalves (dont Anadara notabilis, Codakia orbicularis, Ostrea equestris) et, parfois (Jolly Beach) des strombes (Strombus gigas, S. pugilis). Ce sont, sinon, comme sur les sites de Whitehead's Bluff à Anguilla (Crock et al. 1995) et Hichman's Shell Heap à Nevis (Wilson 1989), des gastéropodes du médiolittoral rocheux (dont Cittarium pica, Acanthopleura granulata, Nerita peloronta) et des herbiers (Turbo castanea, S. gigas), souvent accompagnés par des bivalves (le plus souvent Arca zebra). Le spectre de Baie Orientale 1 se rapproche plutôt de ces deux derniers exemples tandis que celui d'Étang Rouge 1 s'apparente aux « sites à Arca zebra ».

L'étude récente des deux gisements de Saint-Martin apporte donc un jalon supplémentaire dans la connaissance des modes de vie des groupes de la période archaïque des Petites Antilles, du point de vue de la gestion des ressources et, en particulier, des mollusques. Comme sur la majorité des autres sites côtiers, quelques espèces rentables de mollusques ont été exploitées de manière pragmatique et intensive, à des fins artisanales et/ou alimentaires, ce qui a vraisemblablement déterminé l'implantation des sites. Cette forte spécificité des activités - parfois répétées pendant des occupations courtes, assez caractéristique des sites archaïques - et leur variabilité d'un site à l'autre dénotent une gestion organisée des ressources dans l'espace et le temps. Le site de l'intérieur des terres de Plum Piece à Saba (Hofman et Hoogland 2003 ; Hofman et al. 2006), occupé ponctuellement pour l'exploitation de ressources animales et végétales terrestres, vient en 
illustrer un autre pan sans doute complémentaire. Il est à gager que l'exploration des sites archaïques de l'intérieur des terres révélera dans l'avenir d'autres composantes de ces systèmes d'exploitation des ressources sans doute organisés de manière saisonnière sur plusieurs îles. Au sein de ce tableau et de ces stratégies, les mollusques constituaient une ressource importante totalement intégrée. La prise en compte de ce rôle aide donc à appréhender les modes d'occupation des espaces, la mobilité et la gestion territoriale des premiers occupants chasseurs-cueilleurs archaïques.

Homogénéité et particularismes des schémas d'exploitation des premières sociétés saladö̈des de la période céramique : reflets de la cohésion culturelle et économique de nouveaux migrants?

La période céramique débute, aux environs du vi ${ }^{\mathrm{e}}$ siècle av. J.-C., avec la venue depuis le continent sud-américain de sociétés d'horticulteurs potiers identifiées à la série saladoïde (Figure 1) d'après le site vénézuélien éponyme de Saladero sur le Bas Orénoque (Rouse et Cruxent 1963). Migrant originellement depuis le bassin vénézuélien de l'Orénoque (sous-série Ronquinan saladoïde ; Roosevelt 1980, pp. 193-196) vers les Guyanes et la côte nord du Venezuela (sous-série Cedrosan saladoïde ; Rouse 1986, 1992), ces groupes sont ensuite représentés par leurs assemblages céramiques typiques Cedrosan saladoïdes à Trinidad, Tobago, et dans la plupart des îles des Petites Antilles jusqu'à l'extrémité orientale de la République dominicaine (Rouse 1986, 1992, pp. 77-90 ; Veloz Maggiolo 1991 ; Keegan 2000). Les assemblages matériels, notamment céramiques, présentent une grande unité stylistique. Néanmoins, quelques sites anciens (La Hueca à Vieques, Punta Candelero à Puerto Rico, Hope Estate à Saint-Martin, Trants à Montserrat, Morel, Cathédrale et Gare Maritime de Basse-Terre en Guadeloupe) se distinguent par des spécificités stylistiques qui s'écartent du répertoire classique. Souvent associés à une composante Cedrosan saladoöde (Chanlatte Baik et Narganes Storde 1980 ; Hofman et al. 1999 ; Watters et Petersen 1999a ; Bonnissent et al. 2002b), ces ensembles reflètent soit des variations internes au répertoire saladoïde, liées à des unités familiales ou villageoises (Rouse 1986, p. 10, 1989, p. 389, 1992, pp. 89-90 ; Hofman 1993 ; Watters et Petersen 1999a), soit l'existence de groupes proches mais distincts. Ceux-ci se seraient individualisés sur le continent avant la migration vers l'archipel antillais (série Huecoïde) ou lors de la migration vers les îles (sous-série Huecan saladoïde ; Chanlatte Baik et Narganes Storde 1980 ; Rodriguez López 1991 ; Siegel 1991 ; Oliver 1999).

Les débats ne sont pas clos sur le sujet et l'on conserve le terme de saladoïde ancien pour les sites de notre corpus attestant d'un début d'occupation antérieur au I ${ }^{\mathrm{er}}$ millénaire apr. J.-C. (Figures 2, 3a et b). Il s'agit des sites de la Cathédrale et de la Gare Maritime de Basse-Terre en Guadeloupe, fouillés respectivement par Dominique Bonnissent (INRAP Guadeloupe) en 2001 (Bonnissent et al. 2004a) 
et Thomas Romon (INRAP Guadeloupe) en 2005 (Romon et al. 2006). Et des sites saladoïdes anciens et moyens de Radio Antilles et Trants à Montserrat (Watters 1980 ; Petersen 1996; Watters et Petersen 1999b) et de Hope Estate à Saint-Martin (Bonnissent et al. 1997 ; 1998 ; 2002b). Tous ces sites, à l'exception de Radio Antilles, présentent une composante stylistique rapportée au Huecan ou Huecoïde.

Parmi les sites que nous avons étudiés, les données sur les restes de faune vertébrée ont uniquement été publiées pour les sites de Hope Estate et de la Cathédrale de Basse-Terre (Grouard in Bonnissent et al. 1997; 1998 ; 2004 ; les résultats sont publiés pour d'autres sites anciens, tels que Morel et Folle Anse en Guadeloupe, non intégrés à notre corpus; voir Grouard 2001). De ce fait, les données quantitatives des assemblages d'invertébrés ne peuvent pas être ici pondérées par celles des vertébrés et l'on ne discutera que des premiers. Ils révèlent des profils d'exploitation assez proches sur les cinq sites. Malgré des différences marquées de proportions, les spectres ont en commun une majorité de taxons (Figure 5a) prélevés dans deux milieux majeurs (Figures 5b et 6). Il s'agit, d'une part, d'une composante importante, voire dominante, d'invertébrés liés au domaine terrestre plus ou moins littoral sous la forme de crustacés Gécarcinidés et/ou de mollusques fluviatiles (néritines d'eau douce des sites de la Cathédrale et de la Gare Maritime de Basse-Terre; Serrand 2004; Serrand in Romon et al. 2006). D'autre part, de gastéropodes du médiolittoral rocheux dominés par Cittarium pica ou de petites espèces de type nérites, fissurelles, acmées, etc. La collecte de ces dernières n'est pas la plus rentable, mais est aisément réalisée lors du parcours d'espaces accessibles offrant une relative variété de taxons souvent concentrés (chitons, nérites). Les spectres sont complétés par des taxons prélevés dans les herbiers infralittoraux (dont les strombes) et notablement dépourvus ou presque de bivalves (mais les espèces représentées sont les mêmes, dont Codakia orbicularis). L'exploitation se limite donc essentiellement aux zones faisant transition entre domaines marin et terrestre (Figures 5 b et 6 ) : sur la bande médiolittorale avec une collecte à l'avenant des espèces qui y cohabitent, dont la taille relativement peu intéressante (hormis le burgo) impose l'acquisition de grandes quantités; et dans les zones terrestres du littoral et les rivières où l'exploitation prend une forme assez intensive avec la capture de grandes quantités de crustacés et/ou de néritines. Quelques strombes, enfin, sont capturés régulièrement dans les herbiers marins si l'on en croit la quantité d'éléments produits à partir de la coquille de ce taxon (voir infra).

La contribution des invertébrés du domaine terrestre (crustacés ou néritines) se maintient souvent durant l'occupation de ces sites. Elle décroît parfois en fin d'occupation, souvent au profit des taxons du médiolittoral rocheux (dont Cittarium pica) comme sur les sites de Hope Estate (Serrand 2002) et de la Gare Maritime de Basse-Terre (Serrand in Romon et al., 2006). Toutefois, si les crustacés terrestres (et plus généralement les invertébrés liés au domaine terrestre) 


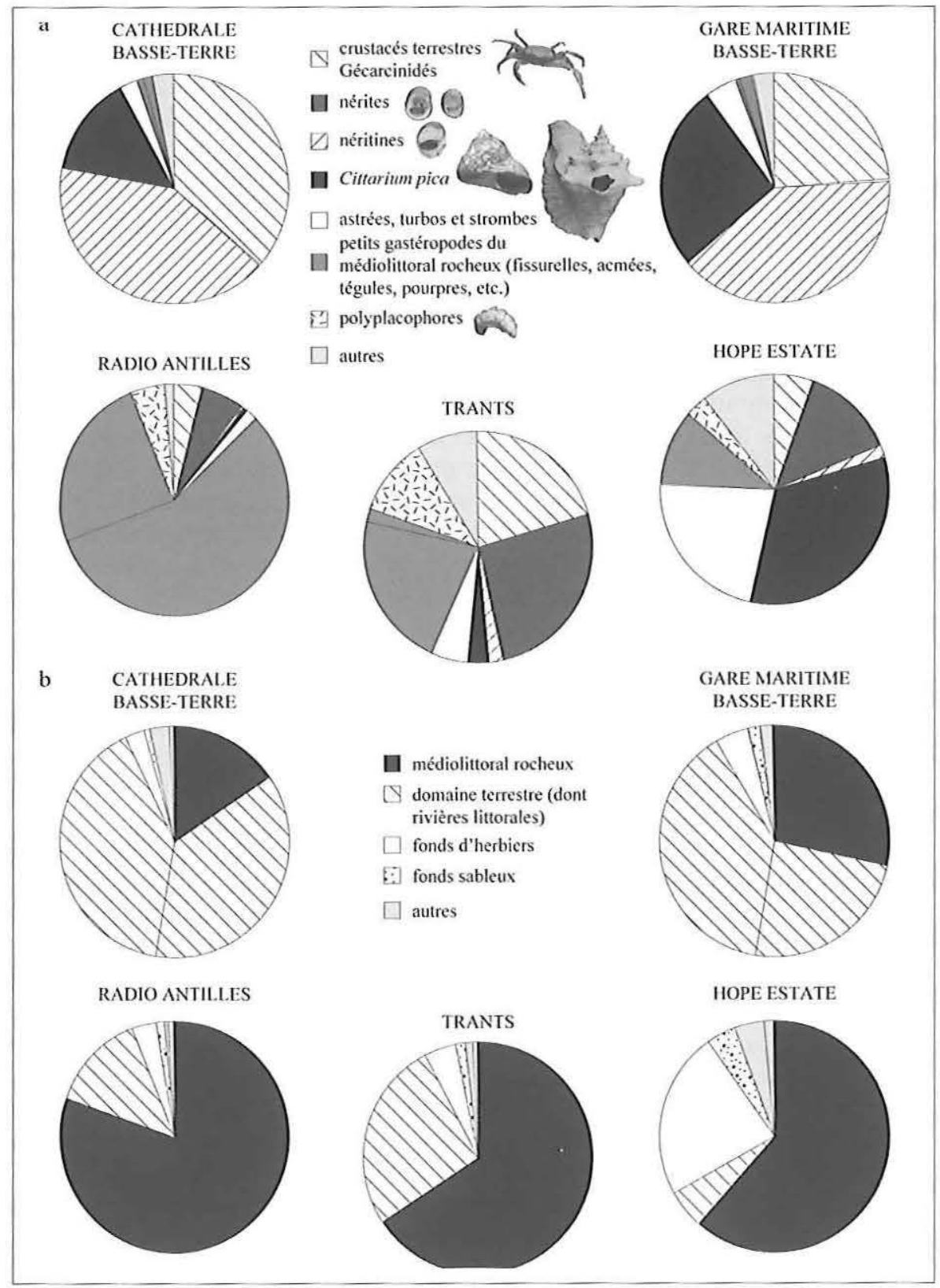

FIG. 5 - Fréquences relatives d'individus ( $\%$ NMI) sur les sites saladoïdes anciens de Cathédrale et Gare Maritime de Basse-Terre (Guadeloupe), Trants et Radio Antilles (Montserrat) et Hope Estate (Saint-Martin) : a. des espèces majeures d'invertébrés exploitées; b. des zones de collecte majeures. 


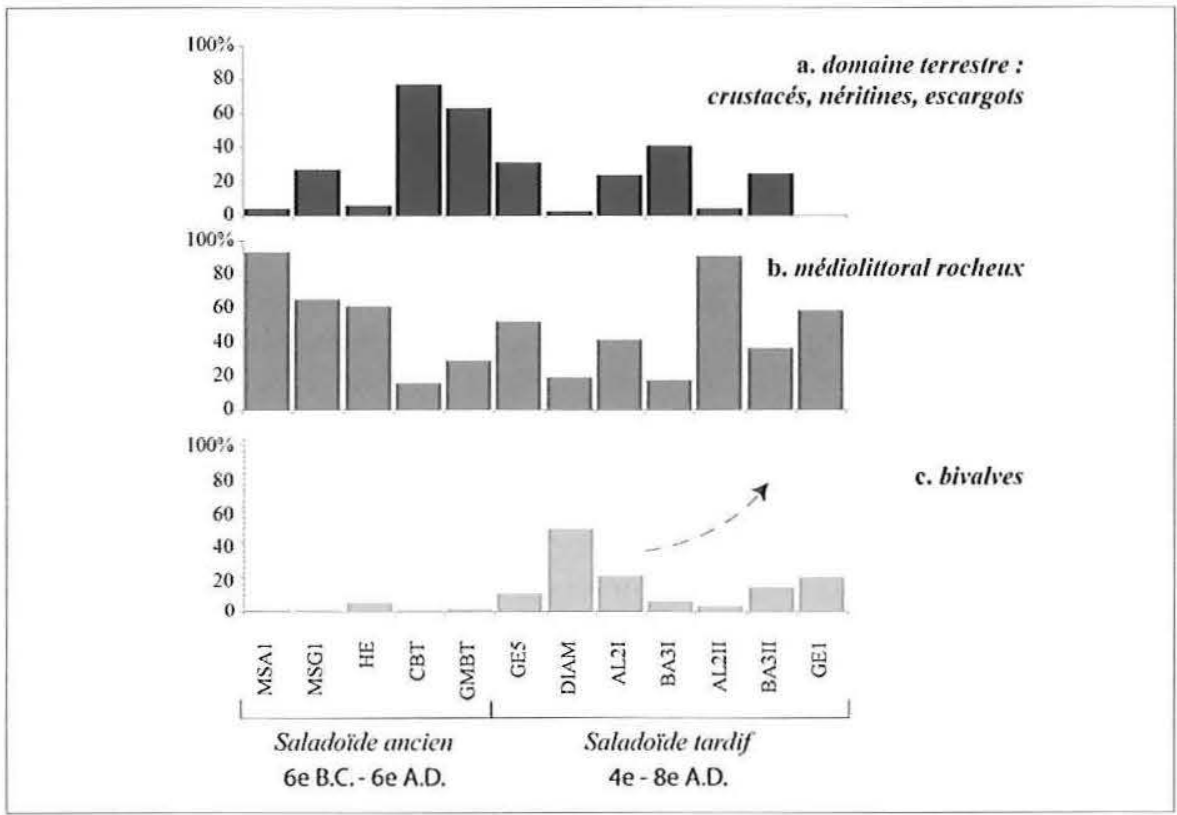

FIG. 6-Représentation schématique de l'évolution sur les sites saladoïdes des Petites Antilles de la part en \% d’individus ( $\%$ NMI) : a. du domaine terrestre ; b. du médiolittoral rocheux ; c. des bivalves.

sont systématiquement exploités sur ces sites saladoïdes anciens, ils ne sont pas toujours dominants (Figure 6) : deux des plus anciens sites, Radio Antilles et Hope Estate, présentent ainsi des fréquences assez moyennes (Serrand 2002). Sur ces deux îles (Montserrat et Saint-Martin), les crustacés sont mieux représentés sur des sites dont l'occupation perdure un peu plus tard (exemple: Trants à Montserrat ; ibid.) ou dont l'implantation est plus tardive et attribuable à la période saladoïde moyenne à récente sans aucun lien avec les spécificités Huecan ou Huecoïde (exemple: Anse des Pères à Saint-Martin; Nokkert 1999). D’ailleurs, la représentation des crustacés terrestres demeure assez élevée dans plusieurs sites tardifs saladoïdes moyens à récents, tous au moins postérieurs au IV $^{\mathrm{e}}$ siècle apr. J.-C. (Figure 6) comme Hichman's à Nevis (Wilson 1989 ; Serrand 2002), Dizac en Martinique (Vidal 1999 ; Serrand 2002, 2005b), Rendezvous Bay à Anguilla (Watters et Petersen 1993 ; Serrand 2002) et Sufferers à Barbuda (Watters 1980 ; Serrand 2002 ; Figure 2). Quoi qu'il en soit, même si les invertébrés liés au domaine terrestre ne sont pas dominants sur tous les sites saladoïdes anciens et qu'ils sont encore exploités sur certains sites postérieurs, leur représentation y est assez systématique (voir aussi les exemples des sites de Morel et Folle 
Anse en Guadeloupe ; Grouard 2001) et plus importante que sur la majorité des sites postsaladoïdes troumassoïdes et suazoïdes (voir infra et Figure 9).

Les sites saladoïdes anciens, éventuellement caractérisés par des composantes céramiques Huecan ou Huecoïde, présentent donc des schémas d'exploitation alimentaire des invertébrés très similaires. Ceux-ci ne sont toutefois pas exclusifs à ces derniers puisqu'ils se maintiennent plus ou moins tardivement dans les sites saladoïdes moyens à récents. En revanche, dans ces sites anciens, de fortes similitudes apparaissent dans l'utilisation des mollusques à des fins artisanales (Figures 7 et 8 ) que l'on ne retrouve pas aussi clairement dans les sites plus tardifs d'Hichman's, Dizac, Rendezvous Bay ou Sufferers (Serrand 2002; 2005b). Si les assemblages de tous ces sites comprennent de nombreuses coquilles de petits gastéropodes (Oliva reticularis, Olivella sp., Conus sp., Cyphoma gibbosum) aménagées pour la parure, des portions de porcelaine Talparia zebra aménagées en outils, des labres de lambi Strombus gigas façonnés en outils et des portions de lambi façonnées en petits éléments apparentés à des " zemis », quelques objets identiques sont propres aux assemblages anciens et semblent révéler un fonds culturel commun très cohérent. Ce sont, en particulier, des portions débitées de gastéropodes, surtout de lambi, et de quelques bivalves transformées selon les mêmes chaînes opératoires en éléments d'ornementation identiques d'un site ancien à l'autre. Tel est le cas des pièces d'incrustation de statuaire en bois de type " dentier », présentes sur les sites de Cathédrale et Gare Maritime de Basse-Terre, Hope Estate et Trants (Figure 7a-g); des portions de Chama macerophylla ou Spondylus sp. façonnées en " goutte » (Gare Maritime, Cathédrale de Basse-Terre et Hope Estate ; Figure 7h-i) ; des plaquettes pendeloques biforées (Cathédrale, Gare Maritime et Hope Estate ; Figure 7j-m) ; des baguettes (Gare Maritime, Hope Estate ; Figure 7n-o) et des représentations zoomorphes, éventuellement reptiliennes (Gare Maritime et Hope Estate; Figure 7p-q). Enfin, la production de perles discoïdes apparaît comme une composante importante sur ces sites saladö̈des anciens (Figure 8). Elle est particulièrement bien représentée sur ceux de la Gare Maritime (1141 pièces) et de Hope Estate (912 pièces) et, dans une moindre mesure, de Trants (202 pièces). Ces ensembles incluent tous les stades de production. Les matières premières sont les mêmes avec l'utilisation essentielle de trois taxons, le lambi Strombus gigas, la chame Chama sarda et le spondyle Spondylus sp. . Les procédés techniques et les chaînes opératoires sont également très similaires ainsi que les produits tant du point de vue morphoscopique que métrique (Figure 8). L'importance de la production de perles discoïdes sur trois des sites saladoïdes anciens et la représentation de tous les stades opératoires suggèrent qu'ils ont été les lieux d'ateliers de production et ont pu jouer un rôle en tant que centres de manufacture, voire de diffusion des produits finis. Les perles discoïdes ne sont pas absentes des sites saladoïdes moyens à récents, mais on les trouve rarement en quantités aussi importantes. 

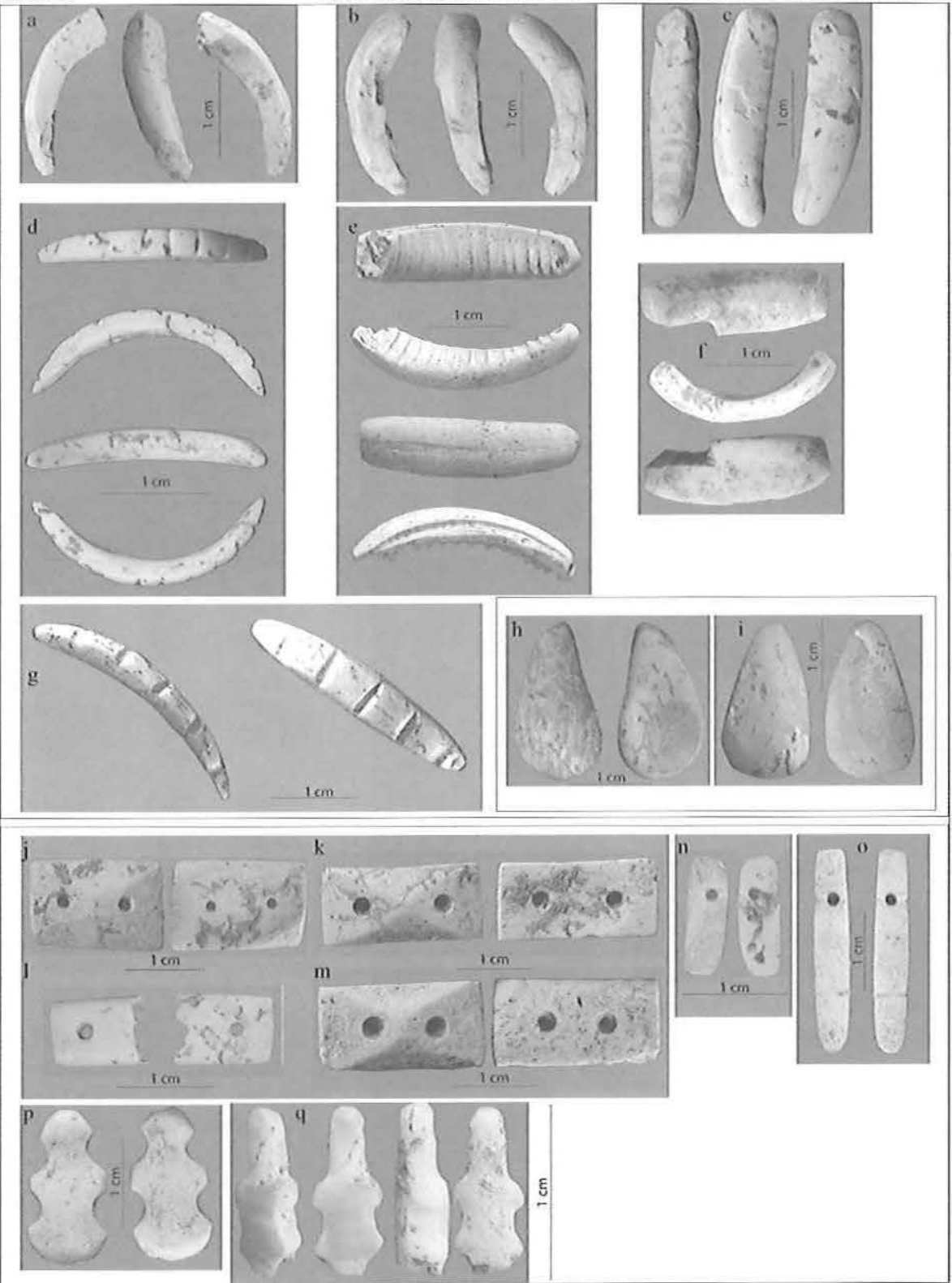

FIG. 7 - Éléments similaires dans le travail de la coquille des sites saladoïdes anciens. Incrustations de statuaire de type «dentier» : a-c. Gare Maritime ; d. Cathédrale de Basse-Terre, Guadeloupe ; e-f. Hope Estate, Saint-Martin ; g. Trants ; éléments de Chama et Spondylus sp. travaillés en "goutte»; h. Gare Maritime; i. Hope Estate; Plaquettes biforées; j. Gare Maritime ; k. Cathédrale ; l-m. Hope Estate ; Baguettes ; n. Gare Maritime ; o. Hope Estate ; Représentations zoomorphes ; p. Gare Maritime ; q. Hope Estate 


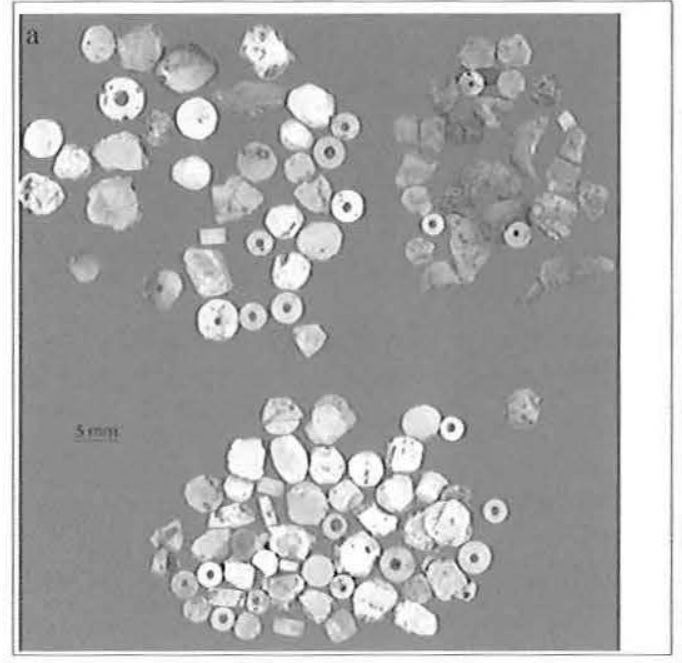

$\mathrm{c}$
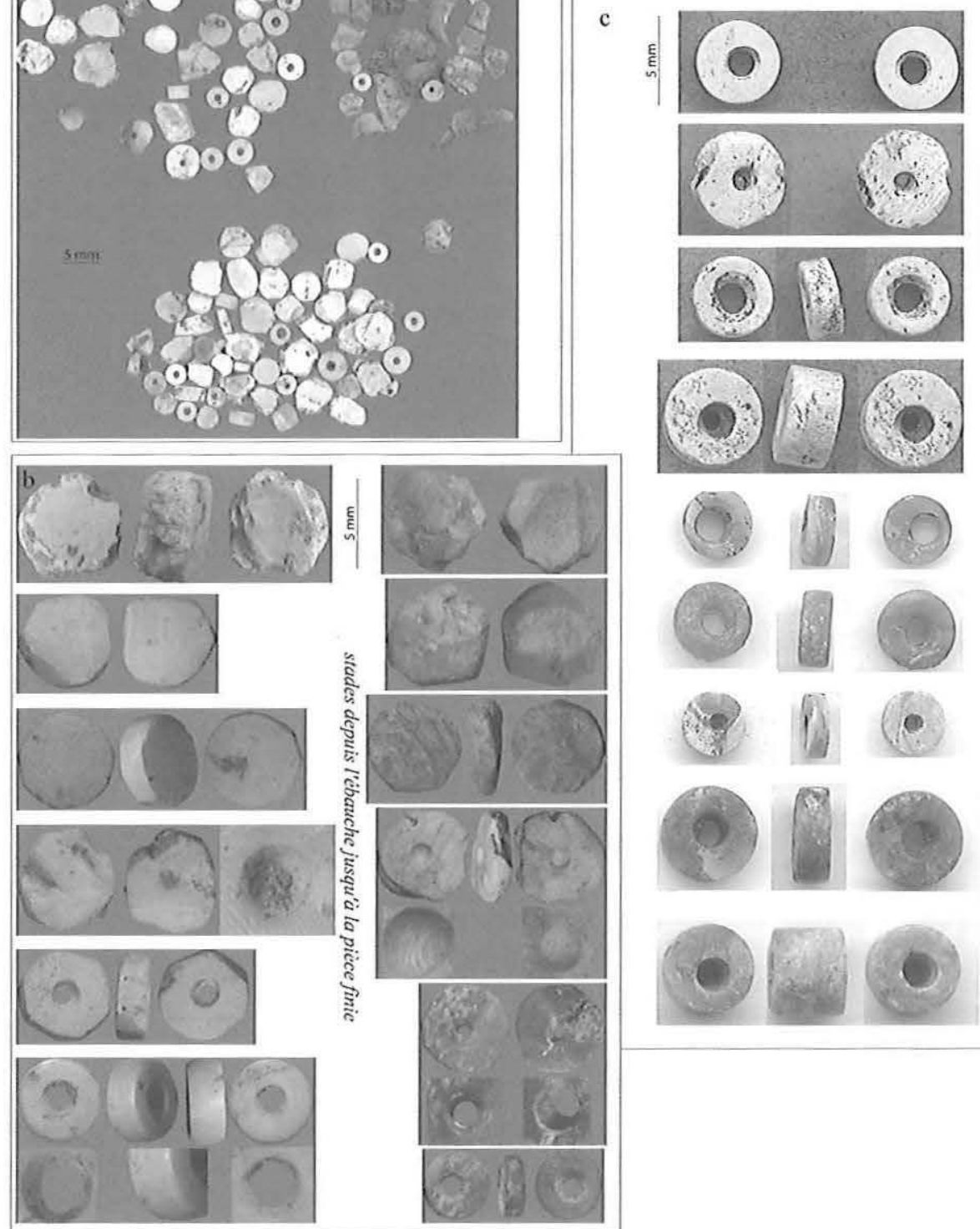

FIG. 8 - Les perles discoïdes des sites saladoïdes anciens de la Gare Maritime de Basse-Terre et Hope Estate : a. exemplaires sur supports divers de la Gare Maritime de Basse-Terre; b. stades de fabrication sur Strombus gigas (gauche) et Chama sarda (droite) à Gare maritime ; c. exemplaires sur Strombus gigas (4 premiers haut), Chama sarda (3 suivants) et Spondylus sp. ( 2 derniers) à Hope Estate 
Les assemblages des sites saladoïdes anciens, en particulier ceux présentant une composante Huecan ou Huecoïde, livrent des assemblages d'invertébrés assez homogènes. Ceux-ci traduisent une exploitation souvent intensive et optimale de taxons du domaine terrestre (crabes, néritines) et une utilisation de mollusques marins restreinte à un ou deux milieux immédiatement accessibles sans être les plus rentables (le médiolittoral est souvent la première zone exploitée lorsque se met en place la collecte de mollusques; Avery et Siegfried 1980) et excluant les autres possibilités d'un large pannel, comme les bivalves. La constance, voire le relatif conservatisme, de ces schémas d'économie (avec des variations sur " le même thème ») chez les premiers groupes ayant migré depuis le continent est peu surprenante. Elle est assez cohérente avec un contexte de colonisation initiale. Ce schéma initial, qui ne se retrouve plus dans les assemblages postérieurs troumassoïdes et suazoïdes des Petites Antilles (voir infra), a perduré plus ou moins tardivement selon les contextes durant la suite de la phase saladoïde (Figure 6), en particulier sur les petites îles comme Nevis, Anguilla ou Barbuda. Mais, sur des îles plus grandes (Martinique notamment) ou plus occidentales (Puerto Rico, Vieques ; Narganes Storde 1985), les données des sites saladoïdes moyens à récents montrent déjà des signes de changement et la mise en place d'une exploitation plus importante des ressources marines, en rapport soit avec une évolution plus rapide de la démographie locale, soit avec la plus grande diversité environnementale de ces îles (Newsom et Wing 2004). Ainsi, sur le site saladoïde moyen à récent de Dizac en Martinique (Serrand 2005b) et sur quelques autres sites (Figure 6), si les crustacés terrestres sont encore bien représentés, de nouvelles composantes, en l'occurrence des bivalves, sont mises à profit. Cette tendance est annonciatrice des schémas d'exploitation de la période postérieure (voir infra).

La mise en évidence de modes d'exploitation des invertébrés et de productions sur coquille assez spécifiques à la phase saladoïde, surtout ancienne, des Petites Antilles participe à la caractérisation progressive des processus, initiaux puis ultérieurs, d'adaptation aux milieux insulaires. Quant à la particularité et à la forte unité des productions sur coquille de cette période, elles devront être précisées par d'autres études afin de voir si l'on peut contribuer aux débats sur la signification culturelle des différents faciès saladoïdes anciens Cedrosan et Huecan.

Grandes tendances des schémas d'exploitation des sociétés saladoïdes tardives, troumassö̈des et suazoüdes : changements de cibles et exploitation intensifiée dans un contexte de diversification culturelle?

Les phases tardives de la période saladoïde, entre les VII ${ }^{\mathrm{e}}$ et $\mathrm{IX}^{\mathrm{e}}$ siècles apr. J.-C. selon les îles, et de la période suivante se caractérisent par une diversification régionale des styles et des assemblages céramiques, des modifications dans la localisation, le nombre et la densité des sites et une expansion de l'occupation à 
tous les espaces insulaires des Antilles (Petites, Grandes Antilles et Bahamas; Figure 1). Ces indices de changement témoignent d'évolutions locales et de mouvements et influences entre les îles et le continent qui produisent une mosaïque d'unités culturelles et socio-politiques plus ou moins complexes, interagissant au travers de réseaux économiques et sociaux (Rouse 1986, 1992 ; Hofman 1993 ; Keegan 2000 ; Delpuech et Hofman 2004). Dans les Grandes Antilles, les assemblages céramiques postsaladoïdes attribués à la série ostionoïde présentent des styles géographiques différents (sous-séries Ostionan et Elenan) qui évoluent de manière divergente avec le temps (sous-séries Meillacan, Chican, assimilables aux sociétés Taïnos des Grandes Antilles, et Palmetto des Bahamas). Dans les Petites Antilles du sud, une série troumassoïde, dérivée du saladoïde tardif, évolue aux environs de 1000 apr. J.-C. en une série suazoïde observée, jusque vers 1500 apr. J.-C. au sud de la Guadeloupe. Dans les Petites Antilles du nord, les assemblages Cedrosan saladö̈des évoluent et se différencient localement en plusieurs styles avec des influences australes troumassoïdes et occidentales ostionoïdes et des liens tardifs probables avec les sociétés Taïnos (Rouse 1992 ; Hofman 1993; Petersen et Crock 1999 ; Crock 2000 ; Delpuech et Hofman 2004).

Ces évolutions culturelles s'accompagnent de changements dans les économies de subsistance. En ce qui concerne les invertébrés, la prépondérance des crustacés terrestres et des gastéropodes du littoral rocheux (voir supra; Serrand 2002), caractéristique des assemblages saladoödes anciens, persiste parfois dans des assemblages saladoïdes tardifs et postérieurs troumassoïdes et suazoïdes, mais n'y est plus aussi systématique (Figure 2). Cette moindre représentation globale des crustacés terrestres (Figure 9a) et, plus généralement, des invertébrés du domaine terrestre dans les périodes suivantes s'accompagne d'une diversification des prises de collecte. Celles-ci fluctuent beaucoup d'un site à l'autre, ce qui semble refléter une plus grande variabilité des formes d'économie. Une seconde tendance apparaît : c'est l'augmentation sensible de l'importance des bivalves (Figure 9). Ils sont plus fréquemment représentés, voire assez souvent dominants, même si cette prépondérance n'est pas systématique et que certains sites postsaladoïdes en sont parfois encore dépourvus.

La moindre représentation des crustacés terrestres est déjà perceptible en fin d'occupation de certains sites saladoïdes anciens et moyens comme Hope Estate (Serrand 2002) et Cathédrale de Basse-Terre (Bonnissent et al. 2004a ; Serrand 2004), mais aussi Indian Creek à Antigua (Jones 1985) ou Sugar Factory Pier à Saint-Kitts (Goodwin 1979). Elle est assez nette dans la majorité des sites saladoïdes tardifs et postérieurs troumassoïdes et suazoïdes (Figures 6a et 9a), malgré quelques exceptions. C'est le cas, dans notre corpus, des sites de transition saladoïde tardif à troumassoïde de Rendezvous Bay à Anguilla et Sufferers à Barbuda, déjà évoqués, et des sites troumassoïdes et suazoïdes d'Indian Castle à Nevis ou de Trabaud en Martinique qui présentent encore des fréquences élevées de crustacés. La tendance globale montre donc que l'exploitation des crustacés 


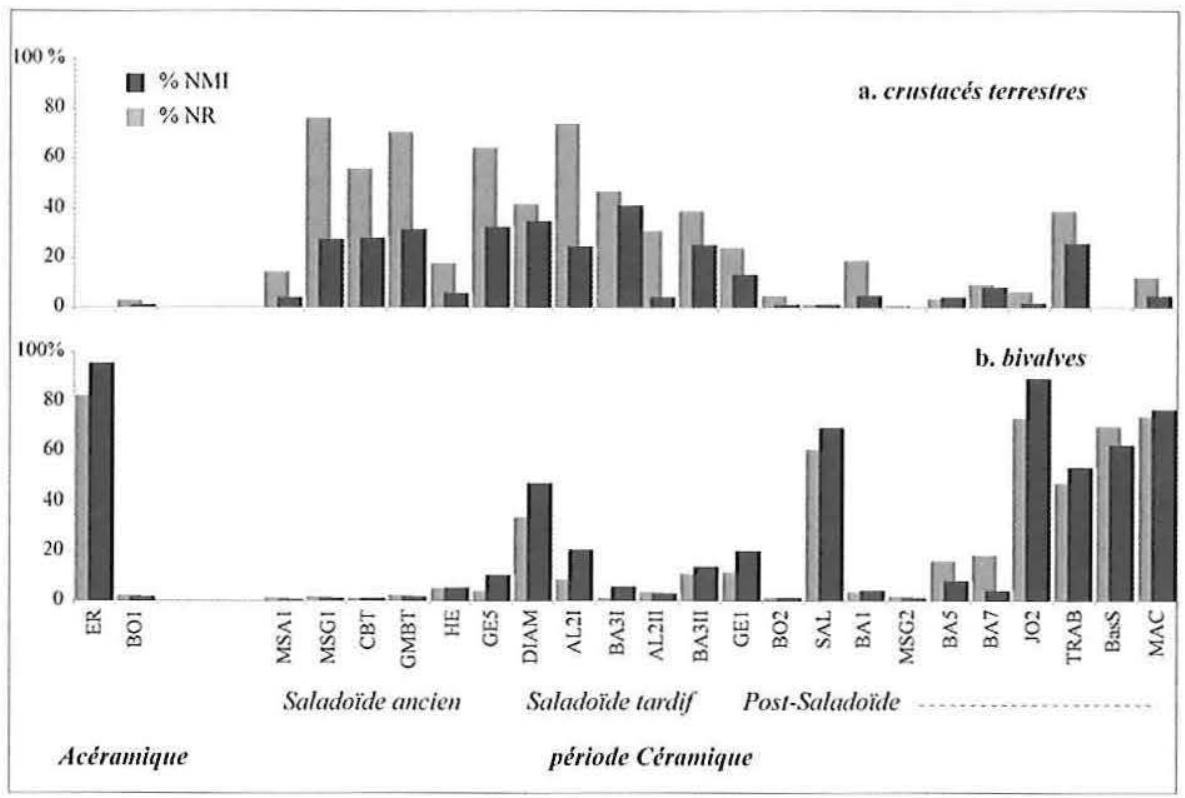

FIG. 9 - Représentation schématique de l'évolution dans le temps sur les sites des Petites Antilles de la part ( $\%$ d'individus NMI et $\%$ de restes NR) : a. des crustacés terrestres ; b. des bivalves

terrestres n'est plus une composante aussi systématique et fondamentale qu'auparavant, mais que ces changements interviennent selon des rythmes et des facteurs variables d'un endroit à l'autre. L'exploitation des crustacés perdure sur certains sites tardifs parce que des populations en font encore une exploitationintensive : c'est sans doute le cas des sites de Rendezvous Bay et Sufferers qui, bien que tardifs, sont les premières implantations connues sur les îles d'Anguilla ou Barbuda, pas ou peu fréquentées avant (voir aussi Pointe du Helleux en Guadeloupe ; Grouard 2001). Ailleurs, notamment dans des îles aux environnements variés, comme Nevis ou la Martinique (Figure 9), certains sites tardifs encore caractérisés par de fortes fréquences de crustacés terrestres peuvent refléter la mise à profit de populations locales encore attractives ou des pratiques spécialisées ponctuelles ou locales, intégrées dans un système d'exploitation de ressources complémentaires à large échelle.

L'augmentation de l'importance des bivalves est, en parallèle, la seconde tendance globale observée au travers des assemblages saladoïdes tardifs et postérieurs, troumassoïdes et suazoïdes, des Petites Antilles, étudiés tant par nousmêmes que par d'autres. Elle est déjà perceptible, en stratigraphie, durant les phases d'occupation les plus tardives de certains sites saladoïdes comme Indian 
Creek à Antigua (Jones 1985) ou Sugar Factory Pier à Saint-Kitts (Goodwin 1979) et nette sur certains sites saladoïdes tardifs comme Anse des Pères à Saint-Martin (Nokkert 1999) ou Dizac en Martinique (Serrand 2005b). Elle est également claire sur plusieurs séquences d'occupation saladoïde/postsaladoïde d'îles comme Antigua (Serrand 2002), Nevis (ibid. ; Figures 3a et b, 10a) ou la Martinique (Serrand s. d. ; Figure 10b ; voir infra) et pour la majorité des sites postsaladoïdes des Petites Antilles. Hors des Petites Antilles, les assemblages saladoïdes tardifs et postérieurs sont également assez systématiquement dominés par des bivalves : c'est le cas sur le site saladoïde tardif et ostionoïde de Paso del Indio à Puerto Rico (Walker 2005) où dominent Anomalocardia brasiliana, Codakia orbicularis et Lucina pectinata et sur plusieurs sites ostionoïdes de Middle Caicos, dans les Bahamas (Keegan 1992). Encore une fois pourtant, malgré cette tendance globale, quelques assemblages font exception comme ceux des sites postsaladoïdes de Barbuda, celui de Windward Bluff à Montserrat et celui de Baie Orientale 2 à Saint-Martin (Figure 9) et, en dehors de notre corpus, une majorité de sites troumassoïdes guadeloupéens comme Anse à la Gourde, Petite Rivière (la Désirade) ou Pointe du Helleux par exemple (voir Nieweg 2000 ; Grouard 2001 ; De Waal 2006). Ces sites, parfois dépourvus de crustacés terrestres, sont encore largement dominés par les taxons du médiolittoral rocheux et ne présentent que de rares bivalves ; certains sont même largement dominés par les crustacés terrestres (voir supra, exemple de la Pointe du Helleux en Guadeloupe). Encore une fois, il est possible que ces sites reflètent des exploitations ponctuellement ou localement spécialisées à l'intérieur de systèmes de sites complémentaires intégrant un éventail plus large de ressources.

Les assemblages fauniques étudiés sur quatre sites (Figure 2) de Martinique (Figure 3a et b; Programme collectif de recherche : Bérard 2003) offrent une intéressante séquence saladoïde moyen ou récent à suazoïde tardif ${ }^{1}$ montrant l'augmentation progressive de l'importance des bivalves (Figure 10b ; Serrand s. d.). Il s'agit des sites saladoïde moyen/récent ( $\mathrm{V}$-VIII ${ }^{\mathrm{e}}$ siècles apr. J.-C.) de Dizac (Vidal 1999), saladoïde moyen/récent et suazoïde ( $\mathrm{VIII}^{\mathrm{e}}$ puis $\mathrm{XII}^{\mathrm{e}}$-XIII ${ }^{\mathrm{e}}$ siècles apr. J.-C.) des Salines (Bérard 2003), troumassoïde tardif et suazoïde ( $\mathrm{xI}^{\mathrm{e}}$ $\mathrm{XIII}^{\mathrm{e}}$ siècles apr. J.-C.) de Trabaud (Allaire 1997) et suazoïde $\left(\mathrm{XII}^{\mathrm{e}}-\mathrm{XV}^{\mathrm{e}}\right.$ siècles apr. J.-C.) de Macabou (Allaire 1981; Grouard et Serrand 2005). Sur cette séquence (Figure 10b), une forte contribution des bivalves (47\% NMI) est déjà sensible sur le site saladoïde moyen/récent de Dizac (Figure 2 ; Serrand 2005b, s. d.) avec, en particulier, Donax denticulatus, petite espèce accessible en bancs denses dont la collecte a été réalisée dans la zone de battement des vagues, au pied du site. L'assemblage des Salines (Figure 2 ; Serrand s. d.) est, lui aussi, largement dominé par les bivalves (69,4\% NMI), en particulier, la chaubette Anomalocardia brasiliana, le donax Donax denticulatus et la praire triangulaire Tivela mactroides. Les bivalves composent de même une grande part (53,4\% NMI) de l'assemblage du site de Trabaud (Figure 2 ; ibid.), avec notamment Lucina pectinata (lucine 

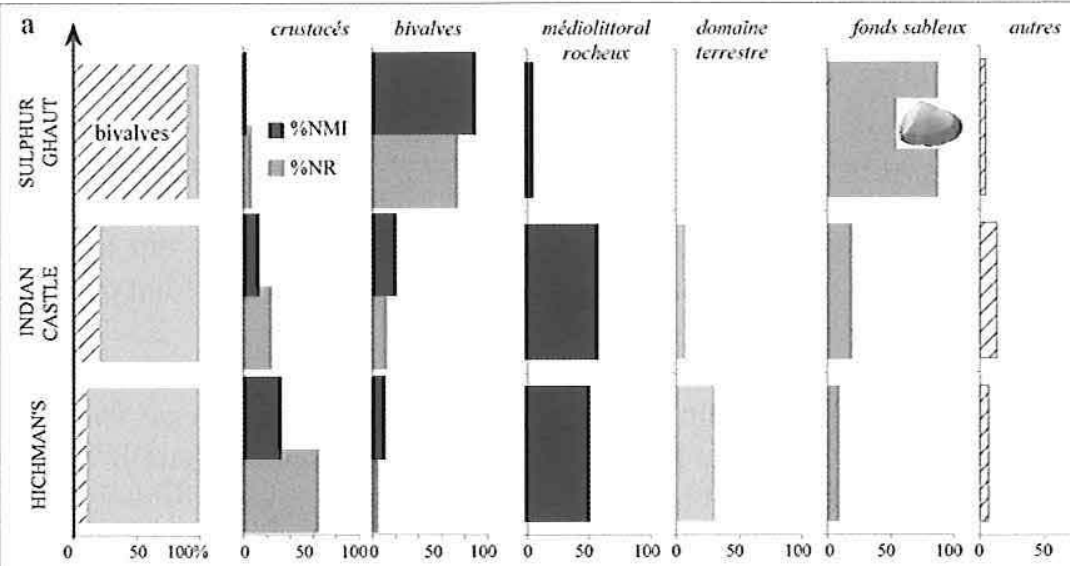

$\%$ NMI

bivalves / autres

$\%$ NR et NMII

crustacés / bivalves

\%NMI zones de collecte
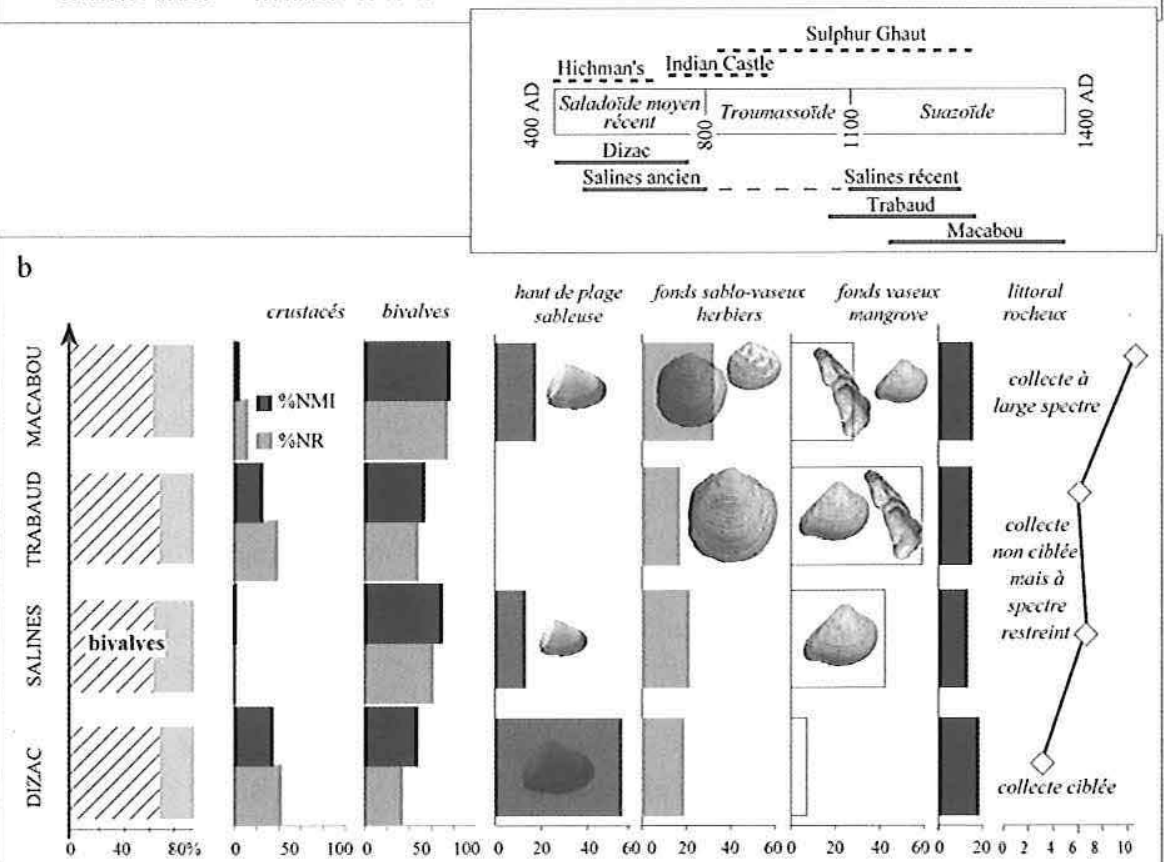

\%NMII

bivalves / autres
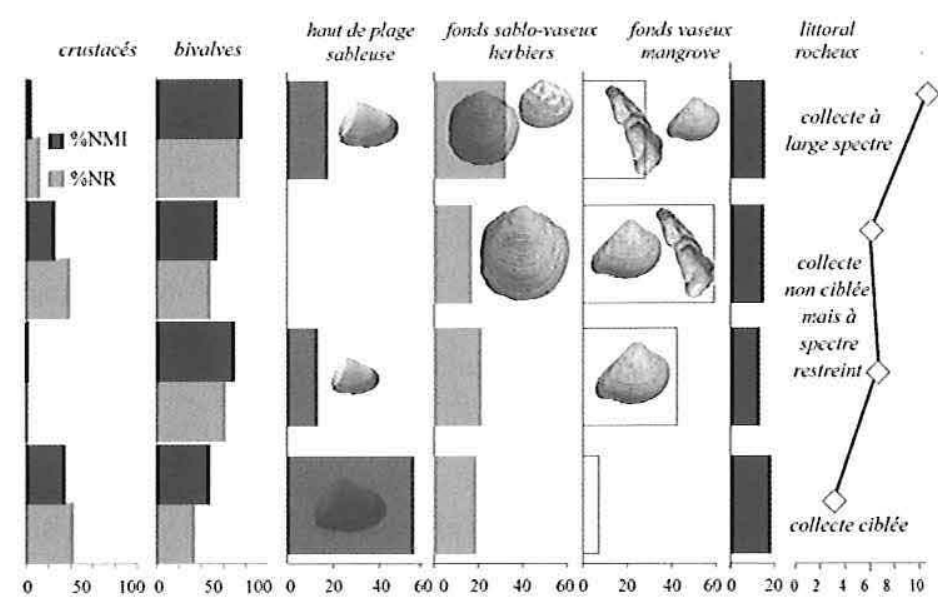

\%NR et NMII
crustacés / bivalves

$\%$ NMIl zones de collecte

Indice de diversité

FIG. 10 - Évolution diachronique sur deux séquences de sites saladoïdes tardifs et post-saladoïdes de la part ( $\%$ d'individus NMI et/ou \% de restes NR) des bivalves, des crustacés versus bivalves et des zones majeures de collecte et espèces associées : a. île de Nevis ; b. Martinique, avec en addition l'évolution de l'indice de diversité des assemblages. 
épaisse), Anomalocardia brasiliana et Crassostrea rhizophorae (huître des palétuviers). Ils dominent largement ( $76,4 \%$ NMI) l'assemblage de Macabou (Figure 2 ; ibid.) avec surtout Donax denticulatus, Crassostrea rhizophorae et Protothaca granulata. Même si l'apport carné que représentent ces bivalves est modeste en regard d'autres ressources (poissons, mammifères), leur présence massive sur les quatre sites révèle le rôle alimentaire important qu'ont joué ces espèces disponibles en grandes densités dans l'environnement immédiat des sites (fonds sableux, vaseux et mangroves).

La séquence de sites martiniquais suggère, par ailleurs, une diversification des prises de la collecte (Figure 10b) avec une évolution de la richesse (nombre d'espèces rapporté à la taille de l'assemblage) et de la diversité (part prise par chacune des espèces) des assemblages. En complément d'une contribution constante, mais moyenne, des zones littorales rocheuses, la collecte est ainsi d'abord ciblée à Dizac sur une espèce et une zone de collecte (battement des vagues); aux Salines et à Trabaud, elle n'est plus ciblée, mais encore restreinte à quelques espèces provenant de deux milieux majeurs (fonds sableux et sablo-vaseux); enfin, à Macabou, les zones et les prises de collecte sont variées et exploitées de manière équilibrée. Cette diversification de la collecte de bivalves dans l'ensemble des environnements disponibles n'est pas observable sur la séquence de sites de Nevis (Figure 10a). Cela peut être dû au petit nombre d'assemblages connus, la contribution des bivalves ne devenant sensible qu'avec le plus tardif, celui de Sulphur Ghaut daté approximativement des $\mathrm{X}^{\mathrm{e}}$-XII ${ }^{\mathrm{e}}$ siècles (et éventuellement contemporain de Trabaud en Martinique). On pourrait donc l'envisager pour la suite de la séquence qui n'est pas, pour l'instant, connue dans cette île.

La meilleure représentation globale des bivalves dans les sites saladoïdes tardifs et surtout postérieurs des Petites Antilles tranche donc avec le schéma observé pour la période antérieure du saladoïde, notamment ancien (voir supra ; Serrand 2002). L'exploitation croissante des bivalves, ressources disponibles en grandes densités, n'exclut pas la collecte d'autres mollusques, comme celle des gastéropodes des zones rocheuses. En revanche, elle va souvent de paire avec la moindre représentation des crustacés terrestres. En Martinique, ces changements interviennent assez clairement en parallèle avec la diversification des lieux d'implantation et la spécialisation fonctionnelle des sites que l'on observe dès le Saladoïde récent et surtout durant la période suazoïde (Bérard 2004 ; Vidal et al. 2004 ; Bérard et al. s. d.). Il est possible que l'intégration croissante des bivalves, taxons disponibles en densités élevées, reflète une exploitation, sinon intensifiée, du moins plus pragmatique, des ressources marines invertébrées. Cela pourrait traduire une adaptation plus grande des systèmes économiques des sociétés postsaladoïdes aux conditions insulaires, peut-être en réponse à l'intensification de l'occupation (observée en Martinique), à des pressions démographiques et/ou à l'éventuel appauvrissement d'autres ressources locales (crabes). Par ailleurs, la moindre homogénéité des schémas d'exploitation (leur point commun réside 


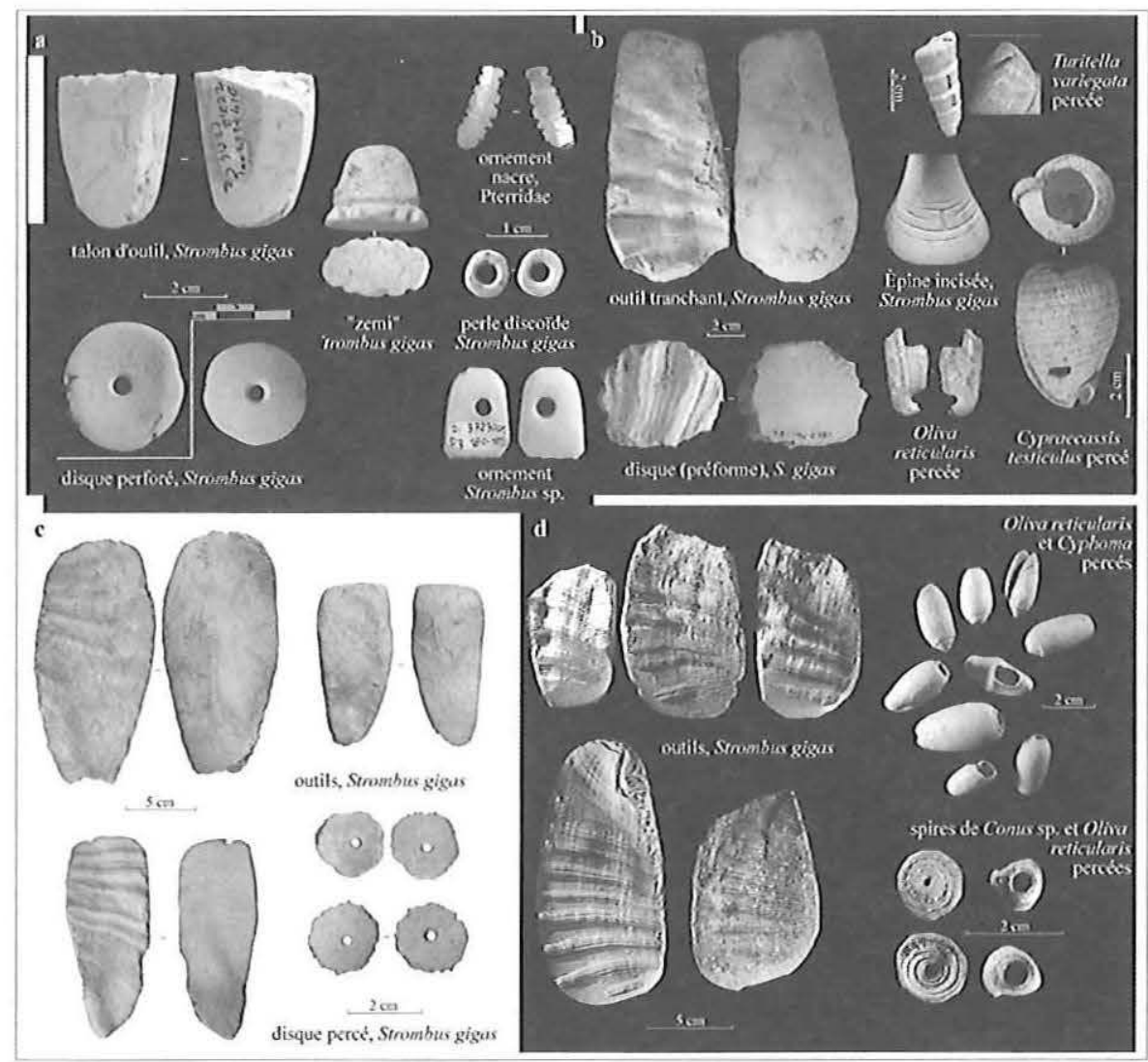

FIG. 11 - Exemplaires d'objets produits sur coquille dans les sites saladoïdes tardifs et post-saladoïdes des Petites Antilles : a. Dizac, Martinique ; b. Macabou, Martinique ; c. Bas de la Source, Guadeloupe ; d. sites de Barbuda : (haut) Indiantown Trail, (bas) Highland Road et Overview Cave.

surtout dans la mise à profit des bancs locaux de bivalves) suggère que les groupes troumassoïdes et suazoïdes, désormais répartis dans une plus grande diversité d'environnements et dans un contexte de diversification culturelle, développent des formes d'économies plus indépendantes. Du point de vue du travail de la coquille, d'ailleurs, les assemblages ne présentent plus la même unité : les productions, si elles dérivent en partie du fond commun saladoïde, sont plus éclectiques d'un site à l'autre (Figure 11).

L'observation des assemblages fauniques répartis sur la séquence précolombienne d'occupation des Petites Antilles septentrionales indique donc d'importantes fluctuations de la contribution des différents invertébrés au cours du temps. 
Particulièrement notables sont celles qui affectent les bivalves et les crustacés terrestres. Les premiers, souvent présents, voire dominants dans les occupations de la période archaïque, sont rares dans les assemblages saladoïdes anciens et à nouveau présents, voire dominants dans de nombreux sites postsaladoïdes (avec des exceptions). En parallèle, les crustacés terrestres, souvent absents des sites de la période archaïque, sont quasiment omniprésents dans les occupations saladoïdes anciennes et nettement moins représentés par la suite, notamment sur les sites postsaladoïdes. Si ces fluctuations peuvent coïncider avec des changements culturels et économiques, le rôle des dynamiques environnementales ne peut être totalement exclu, notamment les modifications de climat et de niveau marin qui auraient pu affecter la disponibilité de ces ressources selon les périodes. Il convient donc de préciser les paramètres naturels autant que les facteurs technoculturels, tous potentiellement en jeu dans l'évolution des économies des groupes amérindiens antillais.

\section{ÉVOLUTION DES SCHÉMAS D'EXPLOITATION DES INVERTÉBRÉS, CADRES NATURELS ET FONCTIONNELS : BEAUCOUP D'INCONNUES}

Les grandes lignes typo-chronologiques de l'occupation précolombienne des Antilles sont assez bien dessinées et permettent de définir les relations dans l'espace et le temps d'entités matérielles, voire culturelles. En revanche, de nombreuses lacunes subsistent encore dans la définition des cadres paléoenvironnemental et socio-économique qui ont entouré l'évolution de ces entités culturelles. L'élaboration précise de ces cadres est pourtant essentielle pour interpréter les données fauniques, les modifications de schémas d'exploitation au cours du temps et, plus généralement, les grandesétapes de l'histoire des cultures amérindiennes.

Du point de vue paléo-environnemental, l'étude d'ensembles archéozoologiques ne fournit qu'un aperçu biogéographique sur les espèces exploitées par l'homme dans des points de l'espace et du temps. Les données paléoenvironnementales plus larges, nécessaires pour compléter cet aperçu, sont encore rares. On méconnaît, en particulier, l'impact potentiel que des changements de climat et de niveau marin pourraient avoir eu sur la disponibilité des différents types de ressources au cours du temps. Or l'existence d'alternances climatiques humides/sèches est suggérée par les rares séquences palynologiques et sédimentologiques établies sur le continent, à Puerto Rico, en Haïti et à Saint Thomas (Carbone 1980 ; Higuera-Gundy 1991 ; Nichols et al. 1977 ; Hodell et al. 1991 ; Curtis et Hodell 1996 ; Burney et al. 1994).

De telles alternances climatiques liées aux déplacements de la zone de convergence intertropicale ont également été mises en évidence très localement sur le site d'Anse à la Gourde en Guadeloupe (Beets et $a l$. 2006), entre les vi ${ }^{e}$ et XII ${ }^{e}$ siècles de notre ère et, à l'échelle de l'île de Saint-Martin, sur les quatre derniers 
millénaires (Bertran et al. 2004 ; Bonnissent et al. 2004b). Des concordances relatives apparaissent à Anse à la Gourde entre les phases d'occupation humaine et les périodes les plus humides (Beets et al. 2006), suggérant l'impact des conditions climatiques sur les stratégies locales d'occupation. À Saint-Martin, des concordances plus larges apparaissent, d'une part, entre la période d'occupation archaïque de l'île jusque vers $800-500$ avant notre ère et une phase sèche à forte activité cyclonique, d'autre part, entre le peuplement de l'île par les sociétés d'horticulteurs saladoïdes et une phase humide située dans le courant du $\mathrm{I}^{\mathrm{er}}$ millénaire de notre ère (Bertran et al. ibid. ; Bonnissent et al. ibid.). Ces données mettent en lumière la variabilité des paléo-environnements durant les phases d'occupation amérindienne des Petites Antilles. Elles en soulignent le rôle potentiel, en complément d'autres facteurs (culturels, démographiques, sociopolitiques et économiques), dans les choix d'économie, d'occupation et de mobilité à des échelles aussi bien réduites (un site, une côte, une partie d'île) que plus larges (grandes étapes d'occupation et d'évolution culturelle amérindiennes).

Les modifications ponctuelles, abruptes ou graduelles, des paléo-environnements - notamment littoraux, en relation avec ces alternances climatiques, les variations holocènes des niveaux marins ${ }^{2}$ (Lighty et al. 1982 ; Digerfeldt et Hendry 1987 ; Feller et al. 1990 ; Tanner 1991 ; Walker et al. 1995 ; Delpuech et al. 2001) et les cycles de perturbations tropicales - ont dû affecter l'accès aux différentes ressources et l'usage que les populations amérindiennes en ont fait (Nicholson 1976 ; Keegan 1999, pp. 95-101), notamment en ce qui concerne les mollusques (Bigalke 1973; Voigt 1975; Meehan 1982; Rollins et al. 1986 ; Keegan et al. 2003). Elles sont susceptibles d'avoir influencé ou modelé certains schémas d'exploitation et types de fonctionnement économique au cours du temps. La question se pose en particulier pour les variations de la contribution des bivalves, dont le rôle prépondérant durant la période archaïque et les phases troumassoïde et suazoïde de la période céramique pourrait avoir un lien avec les conditions climatiques (apparemment plus sèches) qui caractérisent ces périodes.

Outre les méconnaissances encore importantes du cadre paléo-environnemental précolombien, il est aussi délicat d'avoir une vision claire de l'organisation technique et socio-économique (sans parler des systèmes sociopolitiques) propre à chaque grande phase culturelle, voire à chaque groupe de populations. On manque notamment de données sur les modes d'implantation et d'occupation des habitats (permanents ou temporaires, groupés ou dispersés, spécialisés ou non) et sur la gestion des territoires (combinaison de mobilité, sédentarité et itinérance ; existence de réseaux de sites complémentaires ; structuration socio-politique des espaces avec appropriation ethnique de territoires, etc.). Il est ainsi difficile d'identifier des réseaux de sites contemporains à fonctions spécifiques (horticole, sylvicole, halieutique, etc.) qui auraient pu fonctionner de manière complémentaire à l'échelle d'une ou de plusieurs îles. Sans la connaissance de ces systèmes paléo-économiques, on continuera à mal appréhen- 
der les modes de gestion spatiale et temporelle, éventuellement saisonniers, des territoires et des ressources, et l'organisation des activités d'exploitation. Or il est parfaitement vraisemblable que ce type d'organisation complexe ait affecté l'exploitation (collecte et consommation) des mollusques, avec des étapes dissociées dans l'espace et/ou le temps, déterminées en fonction de rythmes saisonniers, de la mobilité des groupes au sein des territoires, de leurs stratégies de déplacement (collecte et consommation peuvent être réalisées de manière expédiente lors de déplacements ; Bigalke 1973 ; Meehan 1982), de l'organisation générale des autres activités d'approvisionnement et de nombreux autres facteurs, y compris, à nouveau, d'ordre paléo-environnemental. L'organisation de l'exploitation des mollusques, dans l'espace et le temps, résulte donc d'interactions entre contingences et phénomènes environnementaux cycliques ou graduels et choix culturels et économiques en relation avec les autres composantes du système socioéconomique. Du fait de cette complexité, un seul assemblage faunique ne rend pas nécessairement compte du système complet d'exploitation de ces ressources. Pour ce faire, il faudrait pouvoir appréhender conjointement le contexte paléoenvironnemantal et le réseau de sites, de ressources et de territoires fonctionnant ensemble, exigence que l'archéologie parvient encore difficilement à satisfaire dans la Caraïbe comme ailleurs (Deith 1986 ; Chenorkian 1992 ; Henshillwood et al. 1994). Les données archéologiques disponibles amènent néanmoins à proposer quelques grandes lignes.

Ainsi, on peut envisager, pour les groupes de la période archaïque, une organisation socio-économique fondée sur une grande mobilité, relayée par des réseaux d'occupations complémentaires dispersées dans un large territoire probablement fréquenté selon une gestion organisée des ressources (éventuellement saisonnière) et des obligations sociales et économiques précises. Les données sur les mollusques suggèrent, de fait, une exploitation maîtrisée et pragmatique de cette ressource, intégrée dans des rythmes de fréquentation des territoires en fonction des besoins spécifiques, alimentaires ou artisanaux. Chaque assemblage faunique ne peut, pour l'instant, être interprété que dissocié des autres, mais pourrait ne représenter qu'un aspect spécifique d'un de ces systèmes économiques ayant fonctionné à l'échelle d'une ou plusieurs îles. Seule l'identification de la fonction relative des sites contemporains (au travers des activités dominantes) et de leurs interrelations permettra d'esquisser de tels réseaux territoriaux et d'appréhender le fonctionnement économique de ces premiers occupants chasseurs-cueilleurs.

Par contraste, les modes de vie de la période céramique ancienne apparaissent plutôt structurés par une relative sédentarité et une économie complète fondée sur l'exploitation de ressources complémentaires au sein d'un territoire large, mais organisé autour de villages à occupation relativement pérenne. La sphère économique est élargie, au-delà, par l'existence de systèmes d'échanges et d'interactions. Les données sur les mollusques suggèrent une exploitation optimale des 
ressources terrestres disponibles et plus timide des ressources marines les plus accessibles sans être les plus rentables (pas d'exploitation de bivalves), schéma assez répandu quelles que soient les contingences/possibilités locales. À cette époque, les assemblages recueillis dans les sites d'habitat seraient assez représentatifs des systèmes globaux d'exploitation (plus centrés sur les villages). On ne maîtrise toutefois pas la part d'exploitation qui a pu se dérouler hors des sites d'habitat majeurs, notamment dans le cadre de déplacements ponctuels ou en parallèle à d'autres activités économiques, où des ressources comme les mollusques, sans doute secondaires, peuvent être consommées de manière expédiente.

Enfin, pour la période céramique plus récente, les modifications observées dans l'emprise territoriale, la multiplication des sites de taille variable et l'apparente spécialisation fonctionnelle des activités sur les sites suggèrent la mise en place de systèmes complexes d'implantations spécialisées, complémentaires voire hiérarchisées, constituant des réseaux économiques, mais aussi socio-politiques. Dans ce cas, il est particulièrement délicat de prétendre reconstituer de manière complète les schémas d'exploitation économique en ne s'appuyant que sur quelques sites qui peuvent, chacun, ne refléter qu'un aspect très spécifique des systèmes paléo-économiques. De plus, dans des espaces naturels parfois déjà largement sollicités durant les phases d'occupation antérieures et dont l'occupation s'intensifie, l'impact humain sur les ressources, variable d'un espace à l'autre selon l'histoire de son occupation, peut être un facteur d'instabilité et un paramètre en jeu dans le choix de stratégies d'exploitation.

\section{Conclusion}

Les modes d'exploitation des invertébrés terrestres et marins par les populations amérindiennes précolombiennes des Antilles ont varié au cours du temps. On n'évalue pas encore pleinement le rôle et l'interaction, dans ces changements, des divers facteurs paléo-environnementaux et socioéconomiques propres aux phases chrono-culturelles archaïques et céramiques. Même si les différentes formes d'exploitation reflètent une part d'adaptation aux possibilités locales des environnements, elles traduisent aussi clairement les options économiques et culturelles adoptées par les différentes sociétés au fil du temps. Au même titre que les autres données archéologiques et combinées avec elles, les observations sur les stratégies d'exploitation des mollusques (subsistance et production) contribuent ainsi à révéler certains traits caractéristiques de l'organisation et de l'évolution culturelles et économiques (mobilité des groupes archaïques ; cohésion culturelle saladoïde ancienne ; expansion territoriale et différentiation culturelle saladoïdes tardives et postsaladoïdes par exemple) des sociétés précolombiennes.

Sans en avoir développé tous les aspects, il apparaît clairement que l'étude archéozoologique et technologique des assemblages de restes d'invertébrés peut contribuer à aborder un ensemble complexe de paramètres environnementaux, 
sociaux, démographiques, économiques et territoriaux. Elle contribue à éclairer l'évolution des milieux naturels, la mise en place et l'adaptation des sytèmes socio-économiques, l'impact des activités humaines sur les ressources ou encore certaines facettes culturelles des sociétés. La démarche suivie impose un accroissement permanent des données et des allers-retours constants entre les informations archéozoologiques fournies par les vestiges fauniques, les données paléoenvironnementales et les schémas paléo-économiques et socio-politiques qui émergent de l'ensemble des autres données archéologiques. *

* Manuscrit reçu en août 2006, accepté pour publication en janvier 2007.

\section{NOTES}

Remerciements : Cet article est la synthèse de travaux menés depuis 1995 avec de nombreux collaborateurs. Qu'ils trouvent ici l'expression de ma reconnaissance et du plaisir à travailler avec eux : Jean-Denis Vigne de l'UMR 5197, CNRS-MNHN, Pierre Lozouet du Laboratoire de biologie des invertébrés marins du MNHN, André Delpuech et Antoine Chancerel respectivement ancien et actuel conservateurs du SRA de Guadeloupe, Christian Stouvenot du SRA Guadeloupe, Olivier Kayser ancien conservateur du SRA de Martinique, Benoît Bérard de l'université Antilles-Guyane, Nathalie Vidal SRA Martinique et SDA, Christophe Henocq de l'association archéologique Hope Estate, Dominique Bonnissent et Thomas Romon de l'INRAP Guadeloupe, David R. Watters du Carnegie Museum of Natural History de Pittsburgh, le regretté James B. Petersen des universités du Maine à Farmington et du Vermont à Burlington, Elizabeth S. Wing, William F. Keegan et Irvy Quitmyer du Museum of Natural History de Floride à Gainesville, Jeff Walker de l'Heritage Resources, Caribbean National Forest de Puerto Rico et Samuel Wilson de l'université du Texas à Austin.

1. Aucune donnée faunique n'est malheureusement disponible sur cette île pour les phases antérieures, les sites étant localisés sur des sédiments acides qui n'ont pas permis la conservation des restes.

2. Parmi les rares études locales sur les fluctuations du niveau marin, les travaux effectués en Guadeloupe (Delpuech et al. 2001) et sur la côte du Golfe de Floride (Tanner 1991 ; Walker et al. 1995) identifient un niveau marin inférieur à l'actuel vers $600 \mathrm{apr}$. J.-C. avec une transgression débutant peut-être dès 600 ou vers 900/1200 apr. J.-C. (Hodell et al. 1991). Toutefois, cette transgression est délicate à appréhender localement du fait des oscillations et de l'activité tectonique propres à chaque île.

\section{BIBLIOGRAPHIE}

Allaire Louis

1981 «Macabou excavations, Martinique: 1972-1979 », Boletín del Museo del Hombre Dominicano, 10 (16), pp. 41-48.

1997 "Anse Trabaud : rapport 1997 », in Jean-Pierre Giraud (éd.), Le Néolithique de la Martinique dans son contexte antillais, Projet collectif de recherche, SRA, Martinique, Fort-de-France.

Armstrong Douglas V.

1978 Archaic shellfish gatherers of St Kitts, Leeward Islands : a case study in subsistence and settlement patterns, unpublished master's thesis, department of Anthropology, UCLA. 
Avery Graham et William R. SIEgFrIed

1980 "Food gatherers along South Africa's seashore ", Oceans, 4, pp. 32-37.

Beets Cees J., Simon R. Troelstra, Pieter M. Grootes, Marie-Josée Nadeau, Klaas van der Borg, Arie F. M. de Jong, Corinne L. Hofman et Menno L. P. Hoogland

2006 "Climate and pre-Columbian settlement at Anse à la Gourde, Guadeloupe, northeastern Caribbean ", Geoarchaeology, 21 (3), pp. 271-280.

BERARd Benoît

2004 Les premières occupations agricoles de l'arc antillais, migration et insularité. Le cas de l'occupation saladö̈de ancienne de la Martinique, BAR series 1299, Monographs in American Archaeology 15, Paris.

BERARd Benoît (éd.)

2003 Le Néolithique martiniquais dans son contexte antillais, rapport de programme collectif de recherche, SRA Martinique, Fort-de-France.

Berard Benoit, Sandrine Grouard et Nathalie SERrand

s. d. "L'occupation post-saladoïde du sud de la Martinique, une approche de l'idée de territoire ", Proceedings of the 21st international Congress of the association for Caribbean archaeology, 2005, International Association for Caribbean Archaeology, Port of Spain, Trinidad.

Bertran Pascal, Dominique Bonnissent, Didier Galop, Daniel Imbert, Pierre Lozouet, Nathalie Serrand et Christian Stouvenot

2004 "Paléoclimat des Petites Antilles depuis 4000 ans BP : l'enregistrement de la lagune de Grand-Case à Saint-Martin », Comptes rendus Géoscience, 336, pp. 1501-1510.

BIGALKE Erich $\mathrm{H}$.

1973 "The exploitation of shellfish by coastal tribesmen of the Transkei », Annals of the Cape Provincial Museum (Natural History), 9, pp. 159-175.

Bonnissent Dominique, Christian Stouvenot, Valérie Boulfroy, François-Xavier Chauviere, Isabelle Geith, Sandrine Grouard, Christophe Henocq et Nathalie Serrand

1997 Fouille programmée du site amérindien de Hope Estate, Saint-Martin, Antilles françaises. Campagne de fouilles 1997, rapport de fouilles programmées au SRA Guadeloupe, Ministère de la Culture, Association archéologique de Hope Estate, Saint-Martin, Marigot, 200 p.

Bonnissent Dominique, Christian Stouvenot, Valérie Boulfroy, François-Xavier Chauviere, Isabelle Geith, Sandrine Grouard, Pascal Listrat et Nathalie Serrand

1998 Fouille programmée du site amérindien de Hope Estate, Saint-Martin, Antilles Françaises. Campagne de fouilles 1998, rapport de fouilles programmées au SRA Guadeloupe, Ministère de la Culture, Association archéologique de Hope Estate, Saint-Martin, 200 p.

Bonnissent Dominique, Pascal Bertran, Antoine Chancerel et Thomas Romon

2001 "Le gisement précéramique de la Baie orientale à Saint-Martin (Petites Antilles). Résultats préliminaires ", in Luc Alofs et Raymundo A. C. F. Dijkhoff (éds), Proceedings of the 19th international Congress of the association for Caribbean archaeology, Publication of the Museo Archeologico, 9, Aruba, pp. 78-88. 
Bonnissent Dominique, Pascal Bertran, Antoine Chancerel, Sandrine Grouard, Thomas Romon, Nathalie Serrand, Christian Stouvenot et Christophe Tardy

2002a Les sites de la Baie Orientale. Occupations précéramiques et post-saladoüdes, Saint-Martin, Guadeloupe, Petites Antilles, document final de synthèse auprès du Service régional d'archéologie de Guadeloupe, Basse-Terre, $131 \mathrm{p}$.

Bonnissent Dominique, Christophe Henocq et Christian Stouvenot

2002b "Le site amérindien de Hope Estate (Saint-Martin, Petites Antilles) : extension et chronologie ", in André Delpuech, Jean-Pierre Giraud et Albert Hesse (éds), Archéologie précolombienne et coloniale des Caraibes, Actes du $123^{e}$ Congrès national des Sociétés historiques et scientifiques, Antilles/Guyane 1998, Éditions du CTHS, Paris, pp. 177-194.

Bonnissent Dominique, Thomas Romon, Pascal Bertran et Nathalie Serrand

2004a Le site de la Cathédrale de Basse-Terre, Guadeloupe, rapport au service régional de l'archéologie de Guadeloupe, Basse-Terre, 269 p.

Bonnissent Dominique, Pascal Bertran, Didier Galop, Daniel Imbert et Christian STOUVENOT

2004b «Chronologie des occupations précolombiennes de l'ìle de Saint-Martin (Petites Antilles) et relations avec les paléo-environnements ", $129^{e}$ Congrès national des sociétés historiques et scientifiques: méthodologie du temps géologique, Besançon, 2004, Editions du CTHS, Paris, pp. 306-307

Bonnissent Dominique, Pascal Bertran, Pierrick Fouere et Nathalie Serrand

2005 Cultures précolombiennes des Petites Antilles. Les occupations précéramiques de l'Étang Rouge 1. Baie Rouge, les Terres Basses, Ile de Saint-Martin, Région Guadeloupe, rapport final de fouilles 2005 auprès du service régional d'archéologie de Guadeloupe, Basse-Terre, 190 p.

BRokKe Alex J.

1999 "Part one : Norman Estate. Shell », in Corinne L. Hofman et Menno L. P. Hoogland (éds), Archaeological investigations on St. Martin (Lesser Antilles). The sites of Norman Estate, Anse des Pères and Hope Estate with a contribution to the "La Hueca problem ", Faculty of Archaeology, Archaeological Studies Leiden University, Leiden, pp. 47-50.

BULLeN Ripley P.

1973 "Krum Bay, a Preceramic workshop on St. Thomas », Proceedings of the 4th international congress for the study of Pre-Columbian cultures of the Lesser Antilles, St. Lucia, 1971, St. Lucia Archacological and Historical Society, Castries, St. Lucia, pp. 110-114.

Burney David A., Lida Pigott Burnrey et Ross D. E.Mchee

1994 «Holocene charcoal stratigraphy from Laguna Tortuguero, Puerto Rico and the timing of human arrival on the island », Journal of Archaeological Science, 21, pp. 273-281.

Callaghan Richard T.

1990 «Possible pre-Ceramic connections between Central America and the Greater Antilles », in Agamemmon Gus Pantel Tekakis, Iraida Vargas 
Arenas et Mario Sanoja Obediente (éds), Proceedings of the 11th international Congress of the association for Caribbean archaeology, Puerto Rico, 1985, La Fundacion Arqueológica, Antropológica e Histórica de Puerto Rico, San Juan, pp. 65-71.

CARbONE Victor A.

1980 "The paleoecology of the Caribbean area ", The Florida Anthropologist, 33 (3), pp. 99-119.

Chanlatte-Baik Luis A. et Yvonne M. Narganes Storde

1980 «La Hueca Vieques : nuevo complejo cultural Agroafarero en la Arqueología Antillana ", in Suzanne M. Lewenstein (éd.), Proceedings of the 8th international Congress for the study of the Pre-Columbian cultures of the Lesser Antilles, St. Kitts 1979, Arizona State University, Arizona State Anthropological Research Papers, 22, Tempe, pp. 501-523.

CHENORKIAN Robert

1992 "Saisonnalisation et malacofaune. Méthodes et possibilités », Préhistoire, Anthropologie Méditerranéennes, 1, pp. 143-168 [LAPMO-CNRS Université de Provence].

Crock John G.

2000 Interisland interaction and the development of chiefdoms in the Eastern Caribbean, Ph. dissertation, University of Pittsburgh, Faculty of Arts and Sciences, Pittsburgh, 347 p.

Crock John G., James B. Petersen et Nick Douglas

1995 "Preceramic Anguilla : a view from the Whitehead's Bluff site », in Ricardo E. Alegria et Miguel Rodríguez (éds), Proceedings of the 15th international Congress of the association for Caribbean archaeology, Puerto Rico 1993, Centro de Estudios Avanzados de Puerto Rico y el Caribe, Fundación Puertorriqueña de las Humanidades y la Universidad del Turabo, San Juan, pp. 283-292.

Curtis Jason H. et David A. Hoddell

1996 "Climate variability on the Yucatan Peninsula (Mexico) during the past 3500 years, and implications for Maya cultural evolution ", Quaternary Research, 46, pp. 37-47.

Davis Dave D.

1982 "Archaic settlement and resource exploitation in the Lesser Antilles : preliminary information from Antigua ", Caribbean Journal of Science, 17 (14), pp. 107-122.

DeFrance Susan D., William F. Keegan et Lee Ann Newsom

1996 " The archacobotanical, bone isotope, and zooarchaeological records from Caribbean sites in comparative perspective ", in Elizabeth J. Reitz, Lee Ann Newsom et Sylvia Scudder (éds), Case studies in environmental archaeology, Plenum Press, New York, pp. 289-304.

DeITH Margaret R.

1986 "Subsistence strategies at a Mesolithic camp site. Evidence of stable isotopes analyses of shells », Journal of Archaeological Science, 1, pp. 119-130. 
Delpuech André, Corinne L. Hofman et Menno L. P. Hoogland

2001 «Excavations at the site of Anse à la Gourde, Guadeloupe. Organisation, history, and environmental setting ", Proceedings of the 18th international Congress of the association for Caribbean archaeology, Grenada 1999, Association internationale d'Archéologie de la Caraïbe, région Guadeloupe, mission archéologique, Grenade, pp. 156-161.

Delpuech André et Corinne L. Hofman (éds)

2004 Late Ceramic Age Societies in the Eastern Caribbean, British Archaeological reports, Monographs in American Archacology, 14, Paris.

DE WAAL Maaike $S$.

2006 Pre-columbian social organisation and interaction interpreted through the study of settlement patterns. An archaeological case study' of the Pointe des Châteaux, La Désirade and Les Iles de la Petite Terre micro-region, Guadeloupe, F.W.I., Department of Caribbean Archaeology, Faculty of Archaeology, Leiden University, Utrecht, 431 p.

Digerfeldt Gunnar et Malcolm D. HeNDRY

1987 "An 8000 year Holocene sea-level record from Jamaica : implications for interpretation of Caribbean reef and coastal history ", Coral Reefs, 5, pp. 165-169.

Feller Christian, Marc Fournier, Daniel Imbert, Claude Caratini et Louis Martin

1990 «Datations ${ }^{14} \mathrm{C}$ et palynologie d'un sédiment tourbeux continu $(0-7 \mathrm{~m})$ dans la mangrove de Guadeloupe (FWI). Résultats préliminaires », Symposium international sur l'évolution des littoraux des Guyanes et de la zone Caraibe méridionale pendant le Quaternaire, 1990, Résumés, ORSTORM, Cayenne, pp. 69-79.

Goomwin Christopher R.

1978 "The Lesser Antillan archaic : new data from St. Kitts ", Journal of the Virgin Islands Archaeological Society, 5, pp. 6-16.

1979 The prehistoric cultural ecology of St. Kitts, West Indies : a case study in island archaeology, unpublished $\mathrm{Ph}$. dissertation, department of Anthropology, Arizona State University, Tempe/University Microfilms, Ann Arbor, $515 \mathrm{p}$.

GrouARD Sandrine

2001 Subsistance, systèmes techniques et gestion territoriale en milieu insulaire antillais précolombien. Exploitation des vertébrés et des crustacés auxépoques saladoüdes et troumassoüdes de Guadeloupe (400 av. J.-C. à 1500 apr. J.-C.), thèse en sciences humaines et sociales, lettres et langues, UFR Sciences Sociales et Administration, Université Paris X, Nanterre, 1073 p.

Grouard Sandrine et Nathalie SERrand

2005 Rapport de fouille programmée du site amérindien de Macabou, rapport au service régional de l'archéologie de Martinique, Fort-de-France, 129 p.

Henshilwood Christopher, Peter Nilsen et John Parkington

1994 "Mussel drying and food storage in the Late Holocene, SW Cape, South Africa ", Journal of Field Archaeology, 21, pp. 103-109. 
Higuera-Gundy Antonia

1991 Antillean vegetational history and paleoclimate reconstructed from the palaeolimnological record of Lake Miragoane, Haiti, Ph. dissertation, University of Florida, Gainesville.

Hodelt. David A., Jason H. Curtis, Glenn A. Jones, Antonia Higuera-Gundy, Mark Brenner, Michael W. Binford et Kathleen T. DORSEY

1991 "Reconstruction of Caribbean climate change over the past 10,500 years ", Nature, 352, pp. 790-793.

Hofman Corinne L.

1993 In search of the Native population of Pre-Columbian Saba (400-1450 A.D.). Part one : pottery styles and their interpretations, unpublished $\mathrm{Ph}$. dissertation, Rijksuniversiteit te Leiden, Leiden, 269 p.

Hofman Corinne L. et Menno L. P. Hoogland

2003 "Plum piece. Evidence for archaic seasonal occupation on Saba, Northern Lesser Antilles around $3300 \mathrm{BP}$ », Journal of Caribbean Archaeology, 4, pp. 12-27.

Hofman Corinne L., Menno L. P. Hoogland et André Delpuech

1999 "New perspectives on a Huecan Saladoid assemblage on Guadeloupe : the case of Morel I ", in Corinne L. Hofman et Menno L. P. Hoogland (éds), Archaeological investigations on St. Martin (Lesser Antilles). The sites of Norman Estate, Anse des Pères and Hope Estate with a contribution to the "La Hueca problem », Faculty of Archaeology, Archaeological Studies Leiden University, Leiden, pp. 303-310.

2001 «Guadeloupe, Saint-François, Anse à la Gourde : fouille programmée pluriannuelle 1995-2000, Rapport de synthèse », University of Leiden, Leiden.

Hofman Corinne L., Alistair J. Bright et Menno L. P. Hoogland

2006 "Archipelagic resource procurement and mobility in the Northern Lesser Antilles : the view from a 3000-year-old tropical forest campsite on Saba ", Journal of Island and Coastal Archaeology, 1, pp. 1-20.

JoNEs Alick R.

1985 «Dietary change and human population at Indian Creek, Antigua », American Antiquity, 50 (3), pp. 518-536.

KEEGAN William F.

1992 The People who Discovered Columbus : the Prehistory of the Bahamas, University Press of Florida, Gainesville, 279 p.

1994 "West Indian Archaeology. 1. Overview and Foragers ", Journal of Archaeological Research, 2 (3), pp. 255-289.

1999 «Recent climatic and sea level fluctuations in relation to West Indian Prehistory ", Proceedings of the 16th international Congress of the association for Caribbean archaeology, Guadeloupe 1995, Conseil régional de la Guadeloupe, Mission archéologique et du Patrimoine, Basse-Terre, Guadeloupe, pp. 95-104.

2000 "West Indian archaeology. 3. Ceramique Age », Journal of Archaeological Research, 8 (2), pp. 135-167. 


\section{Kefgan William F., R. W. Portell et John Slapcinsky}

2003 «Changes in invertebrate taxa at two pre-Columbian sites in southwestern Jamaica, AD 800-1500 ", Journal of Archaeological Science, 30, pp. 1607 1617.

\section{KNIPPENBERG Sebastiaan}

1999 Part one : methods and strategies, in Corinne L. Hofman et Menno L. P. Hoogland (éds), Archaeological investigations on St. Martin (Lesser Antilles). The sites of Norman Estate, Anse des Pères and Hope Estate with a contribution to the "La Hueca problem ", Archaeological Studies Leiden University, Faculty of Archaeology, Leiden, pp. 25-34.

2006 Stone artefact production and exchange among the Northern Lesser Antilles, Leiden University, Faculty of Archaeology, department of Caribbean Archaeology, Utrecht, $382 \mathrm{p}$.

Kozlowski Janusz

1980 "In search of the evolutionary pattern of the Preceramic cultures of the Caribbean ", Boletín del Hombre Dominicano, 9 (13), pp. 61-79.

Lighty Robin G, Ian G. Macintyre et Robert StuckenRath

1982 "Acropora palmata reef framework : a reliable indicator of sea level in the Western Atlantic for the past 100000 years ", Coral Reefs, 1, pp. 125-130.

LUNDBERG Emily R.

1989 Preceramic procurement patterns at Krum Bay, Virgin Islands, unpublished $\mathrm{Ph}$. dissertation, University of Illinois, Urbana-Champaign/University Microfilms, Ann Arbor.

MeEhan Betty

1982 Shell Bed to Shell Midden, Globe Press, Australian Institute of Aboriginal Studies 37, Melbourne, $189 \mathrm{p}$.

Meggers Betty J. et Clifford Evans

1978 "Lowland South America and the Antilles ", in Jesse D. Jennings (éd.), Ancient native Americans, Freeman, San Francisco, pp. 543-591.

NARGANeS STORDE Yvonne M.

1985 "Restos faunísticos vertebrados de Sorcé, Vieques, Puerto Rico », Proceedings of the 10th international Congress for the study of the Pre-Columbian cultures of the Lesser Antilles, Martinique 1983, Centre de recherches Caraïbes, Montréal, pp. 251-264.

Newsom Lee Ann

1993 Native West Indian plant use, Ph. dissertation, department of anthropology, University of Florida, Gainesville/University of Microfilms, Ann Arbor, Michigan.

Newsom Lee Ann et Elizabeth S. WiNG

2004 On land and sea. Native American uses of biological resources in the West Indies, The University of Alabama Press, Tuscaloosa/London, 323 p. 
Nichols Maynard, Edward L. Towle, Golbert Cintron, David GrigG, Robert HuggetT, David Olsen, William Rainey, Edwin Rosenberg, Galen Thompson et Richard Trotman 1977 "Water, sediments and ecology of the Mangrove Lagoon and Benner Bay, St Thomas ", Technical Report, 1, pp. 1-159.

\section{Nicholson Desmond V.}

1976 "The importance of sea level changes in Caribbean archaeology ", Journal of the Virgin Islands Archaeological Society, 3, pp. 19-23.

NiEweg Denis

2000 Shells in archaeology. Archaeological shell on Trinidad and Guadeloupe : two case studies, unpublished MA thesis, Leiden University.

NODINE Bruce K.

1990 "Aceramic populations in the Lesser Antilles : evidence from Antigua, West Indies ", Paper presented at the 55th Anmual Meeting of the Society for American Archaeology, Las Vegas.

NOKKERT Mark

1999 "Part two : Anse des Pères. Faunal exploitation », in Corinne L. Hofman et Menno L. P. Hoogland (éds), Archaeological investigations on St. Martin (Lesser Antilles). The sites of Norman Estate, Anse des Pères and Hope Estate with a contribution to the "La Hueca problem ", Faculty of Archaeology, Archaeological Studies Leiden University, Leiden, pp. 111-125.

Oliver José R.

1999 "The "La Hueca problem" in Puerto Rico and the Caribbean : old problems, new perspectives, possible solutions ", in Corinne L. Hofman et Menno L. P. Hoogland (éds), Archaeological investigations on St. Martin (Lesser Antilles). The sites of Norman Estate, Anse des Pères and Hope Estate with a contribution to the "La Hueca problem », Faculty of Archaeology, Archaeological Studies Leiden University, Leiden, pp. 253-296.

Ortiz Aguilú Juan José, José Rivera Meléndez, Jacome A. Principe, Marisol MélenDez Maiz et M. LAVERGNe Colberg

1991 "Intensive agriculture in precolumbian West Indies : the case for terraces ", in Alissandra Cummins et Philippa King (éds), Proceedings of the 14th international Congress of the association for Caribbean archaeology, Barbados 1991, Barbados Museum and Historical Society, St. Ann's Garrison, Barbados, pp. 278-285.

Petersen James B.

1996 "Archaeology of Trants, Montserrat. Part 3. Chronological and settlement data ", Annals of Carnegie Museum, 65 (4), pp. 323-361.

1997 «Taino, Island Carib, and prehistoric Amerindian economics in the West Indies : tropical forest adaptations to island environments ", in Samuel M. Wilson (éd.), The Indigenous people of the Caribbean, University Press of Florida, Gainesville, pp. 118-130.

Petersen James B. et John G. Crock

1999 "Late Saladoid to late prehistoric occupation in Anguilla : site setting, chronology and settlement hierarchy ", Proceedings of the 18th international 
Congress of the association for Caribbean archaeology, Grenada 1999, vol. 1, Association internationale d'Archéologie de la Caraïbe, région Guadeloupe, mission archéologique, Grenade, pp. 124-135.

RoDríguez López Miguel

1991 «Arqueología de Punta Candelero, Puerto Rico », in Edwin N. Ayubi et Jay B. Haviser (éds), Proceedings of the 13th international Congress of the association for Caribbean archaeology, Curaçao 1989, Institute of the Netherlands Antilles, Reports of the Archaeological-Anthropological Institute of the Netherlands Antilles 9, Curaçao, pp. 605-627.

Rollins Harold B., Daniel H. SANDiveiss et Judith C. Rollins

1986 "Effect of the 1982-1983 El Niño on bivalve mollusks ", National Geographic Research, 2, pp. 106-112.

Romon Thomas, Pascal Bertran, Axel Daussy, Pierrick Fouere, Christine Foullloud, Sandrine Grounrd, Mickaël Mestre, Nathalie Serrand et Christian Valette

2006 Le site de la Gare Maritime de Basse-Terre, Guadeloupe, rapport au service régional de l'archéologie de Guadeloupe, Basse-Terre.

Roosevelt Anna C.

1980 Parmana : prehistoric maize and manioc subsistence along the Amazon and Orinoco, Academic Press, Studies in Archaeology, New York.

Rouse Irving

1986 Migrations in Prehistory: inferring population movement from cultural remains, Yale University Press, New Haven.

1989 "Peopling and repeopling of the West Indies », in Charles A. Woods (éd.), Biogeography of the West Indies, past, present and future, Sandhill Crane Press, Gainesville, pp. 119-136.

1992 The Tä̈ns: rise and decline of the people who greeted Columbus, Yale University Press, New Haven.

Rouse Irving et José CRUXENT

1963 Venezuelan archaeology, Yale University Press, Caribbean Series 6, New Haven.

SERrAnd Nathalie

2002 Exploitation des invertébrés marins et terrestres par les populations saladö̈des et post-saladoüdes du Nord des Petites Antilles (500 av. J.-C.-1200 apr. J.-C.). Étude de cas et comparaisons, thèse de doctorat de l'Université Paris I Panthéon-Sorbonne, Préhistoire-Ethnologie-Anthropologic, Paris.

2004 "Les restes de mollusques du site de la Cathédrale de Basse-Terre, Guadeloupe ", in Dominique Bonnissent et al., Le site de la Cathédrale de BasseTerre, Guadeloupe, rapport au service régional de l'archéologie de Guadeloupe, Basse-Terre, pp. 45-60.

2005a «Exploitation des mollusques et crustacés sur le site précéramique d'Étang Rouge, Saint-Martin, Guadeloupe ", in Dominique Bonnissent et al., Cultures précolombiennes des Petites Antilles. Les occupations précéramiques de l'Étang Rouge 1. Baie Rouge, les Terres Basses, Ile de Saint-Martin, Région 
Guadeloupe, rapport final de fouilles 2005 auprès du service régional d'archéologie de Guadeloupe, Basse-Terre, pp. 106-132.

2005 b «Les restes de mollusques du site saladoïde moyen-tardif du Diamant à Dizac, Martinique (450-700 apr. J.C.) : une exploitation entre mer et mangrove. Résultats préliminaires », in Glenis Tavárez María et Manuel A. García Arévalo (éds), Proceedings of the 20th international Congress of the association for Caribbean archaeology, République Dominicaine 2003, International Association for Caribbean Archaeology, Museo del Hombre Dominicano, Fundación García Arévalo, Santo Domingo, pp. 159-168.

s. d. «L'exploitation des invertébrés durant l'occupation Céramique tardive du Sud de la Martinique : aperçu diachronique au travers des sites de Dizac, Salines, Trabaud et Macabou ", Proceedings of the 21st international Congress of the association for Caribbean archaeology, Trinidad 2005, International Association for Caribbean Archacology [sous presse].

SERrand Nathalie et Dominique Bonnissent

2005 «Pre-columbian Preceramic shellfish consumption and shell tool production: shell remains from Orient Bay, Saint-Martin, Northern Lesser Antilles ", in Daniella E. Bar-Yosef (éd.), Archaeomalacology : molluscs in former enviromment of human behaviour: Proceedings of the 9th ICAZ Conference, Durham 2002, Oxbow Books, Durham, pp. 29-39.

\section{Siegel Peter E.}

1991 "Migration research in Saladoid archaeology : a review », The Florida Anthropologist, 44 (1), pp. 79-91.

Stoner Allan W. et Janice M. WAIte

1990 "Distribution and behavior of Queen Conch, Strombus gigas, relative to seagrass standing crop ", Fishery Bulletin, 88, pp. 573-585.

TANNER William F.

1991 "The "Gulf of Mexico" late Holocene sea level curve and river delta history ", Gulf Coast Association of Geological Societies Transactions, 41, pp. 583-589.

\section{Veloz Maggiolo Marcio}

1991 Panorama histórico del Caribe precolombino, Ed. Banco Central de la República Dominicana, Santo Domingo.

Veloz Maggiolo Marcio et Bernardo Vega

1982 "The Antillean Preceramic : a new approximation ", Journal of New World Archaeology, 5 (1), pp. 33-44.

Versteeg Aad H., Kees Schinkel et Samuel M. Wilson

1993 "Large-scale excavation versus surveys : examples from Nevis, St. Eustatius and St. Kitts in the Northern Caribbean ", Analecta Praehistoria Leidensia, 26, pp. 139-161.

VIDAL Nathalie

1999 "Le site précolombien de la plage Dizac au Diamant, Martinique », Proceedings of the 16th international congress of the association for Caribbean 
archaeology, Guadeloupe 1995, Conseil Régional de la Guadeloupe, Mission Archéologique et du Patrimoine, Basse-Terre, Guadeloupe, pp. 7-16.

VIDAL Nathalie, Benoît Berard et Olivier Kayser

2004 "En vue de l'étude de l'occupation post-saladoïde de la Martinique », in André Delpuech et Corinne L. Hofman (éds), Late Ceramic Age Societies in the Eastern Caribbean, British Archaeological reports, Monographs in American Archaeology 14, Paris, pp. 195-204.

Voigt Elizabeth A.

1975 «Studies of marine mollusca from archaeological sites : dietary preferences, environmental reconstructions and ethnological parallels ", in Anneke Clason (éd.), Archaeozoological Studies, Elsevier, Amsterdam, pp. 87-98.

WALKER Jeff

2005 "The Paso del Indio site, Vega Baja, Puerto Rico : a progress report ", in Peter E. Siegel (éd.), Ancient Borinquen : archaeology and ethohistory of Native Puerto Rico, University of Alabama Press, Tuscaloosa, pp. 55-87.

Walker Karen J., Frank W. Stapor Jr et William H. MarquardT

1995 «Archaeological evidence for a 1750-1450 BP higher-than-present sea level along Florida's Gulf coast », Journal of Coastal Research Special Issue, 17, Holocene cycles : climate, sea levels, and sedimentation, pp. 205-218.

WATTERS David R.

1980 Transect Surveying and Prehistoric Site Locations on Barbuda and Montserrat, Leeward Islands, West Indies, unpublished Ph. dissertation, department of anthropology, University of Pittsburgh, Pittsburg/University Microfilms, Ann Arbor, 416 p.

Watters David R. et James B. Petersen

1993 "Preliminary report on the archaeology of the Rendezvous Bay Site, Anguilla ", in Alissandra Cummins et Philippa King (éds), Proceedings of the 14th international Congress of the association for Caribbean Archaeology, Barbados 1991, Barbados Museum and Historical Society, Barbados, pp. 25-33.

1999a "Is La Hueca style pottery present at Trants? », in Corinne L. Hofman et Menno L. P. Hoogland (éds), Archaeological investigations on St. Martin (Lesser Antilles). The sites of Norman Estate, Anse des Pères and Hope Estate with a contribution to the "La Hueca problem ", Faculty of Archaeology, Archaeological Studies Leiden University, Leiden, pp. 299-302.

1999b "Spatial analysis at Trants, Montserrat ", in Ricardo E. Alegria et Miguel Rodríguez (éds), Proceedings of the 15th international Congress of the association for Caribbean archaeology, San Juan 1995, Centro de Estudios Avanzados de Puerto Rico y el Caribe, Fundación Puertorriqueña de las Humanidades y la Universidad del Turabo, San Juan, pp. 15-25.

2001 «Preliminary report on the correlation of Archaic-Age localities with a paleoshoreline on Barbuda ", in Luc Alofs et Raymundo A. C. F. Dijkhoff (éds), Proceedings of the 19th international Congress of the association for Caribbean archaeology, Publication of the Museo Arqueologico, 9, Aruba, pp. 102-109. 
WiLson Samuel M.

1989 "The prehistoric settlement pattern of Nevis, West Indies », Journal of Field Archaeology, 16 (4), pp. 427-450.

Wilson Samuel M., Harry B. ICeland et Thomas R. Hester

1998 "Preceramic connections between Yucatan and the Caribbean ", Latin American Antiquity, 9 (4), pp. 342-352. 